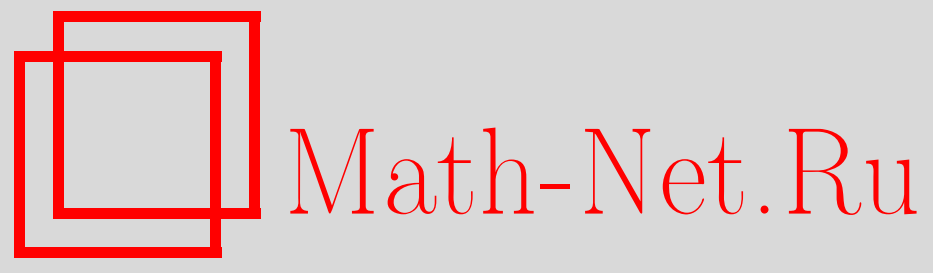

Т. Г. Эргашев, Потенциалы для трехмерного эллиптического уравнения с одним сингулярным коэффициентом и их применение, Вестн. Сам. гос. техн. ун-та. Сер. Физ.-мат. науки, 2021, номер 2, 257-285

DOI: https://doi.org/10.14498/vsgtu1810

Использование Общероссийского математического портала MathNet.Ru подразумевает, что вы прочитали и согласны с пользовательским соглашением

http://www . mathnet.ru/rus/agreement

Параметры загрузки:

IP : 3.85 .183 .62

26 апреля 2023 г., 11:38:25

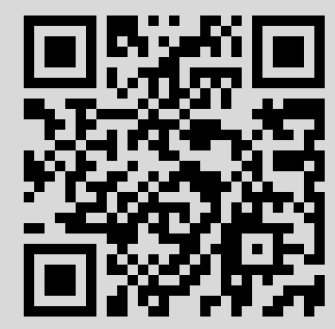


УДК 517.956.6

\title{
Потенциалы для трехмерного эллиптического уравнения с одним сингулярным коэффициентом и их применение
}

\section{(C) T. Г. Эргашев ${ }^{1,2}$}

1 Институт математики имени В. И. Романовского

Академии наук Республики Узбекистан,

Узбекистан, 100174, Ташкент, ул. Университетская, 4-а.

2 Ташкентский институт инженеров ирригации и механизации сельского хозяйства,

Узбекистан, 100000, Ташкент, ул. Кары-Ниязи, 39

\begin{abstract}
Аннотация
Рассматривается теория потенциала для трехмерного эллиптического уравнения с одним сингулярным коэффициентом. В рассмотрение вводятся потенциалы двойного и простого слоев с неизвестной плотностью, которые выражаются через фундаментальное решение названного эллиптического уравнения. При исследовании этих потенциалов используются свойства гипергеометрической функции Гаусса.

Доказаны теоремы о предельных значениях введенных потенциалов и их конормальных производных, которые позволяют эквивалентным образом свести краевые задачи для сингулярных эллиптических уравнений к интегральному уравнению второго рода, к которому применима теория Фредгольма.

В качестве приложения изложенной теории в области, ограниченной координатной плоскостью $x=0$ и поверхностью Ляпунова при $x>0$, для трехмерного эллиптического уравнения с одним сингулярным коэффициентом решается задача Хольмгрена. Единственность решения поставленной задачи доказывается известным методом $a b c$, а существование - методом функции Грина, регулярная часть которой ищется в виде потенциала двойного слоя с временно неизвестной плотностью. Решение задачи Хольмгрена находится в виде, удобном для дальнейших исследований.
\end{abstract}

Ключевые слова: трехмерное эллиптическое уравнение с одним сингулярным коэффициентом, фундаментальные решения, теория потенциала, функция Грина, задача Хольмгрена.

Получение: 22 июля 2020 г. / Исправление: 4 февраля 2021 г. / Принятие: 11 мая 2021 г. / Публикация онлайн: 11 июня 2021 г.

\section{Научная статья}

( @) Ко Контент публикуется на условиях лицензии Creative Commons Attribution 4.0 International (https://creativecommons.org/licenses/by/4.0/deed.ru)

\section{Образец для цитирования}

Эргашев Т. Г. Потенциалы для трехмерного эллиптического уравнения с одним сингулярным коэффициентом и их применение // Вестн. Сам. гос. техн. ун-та. Сер. Физ.-мат. науки, 2021. Т. 25, № 2. C. 257-285. https://doi.org/10.14498/vsgtu1810.

\section{Сведения об авторе}

Тухтасин Гуламжанович Эргашев (10) https://orcid.org/0000-0003-3542-8309 доктор физико-математических наук; лаб. дифференциальных уравнений и их приложений ${ }^{1}$; доцент; каф. высшей математики ${ }^{2}$; e-mail: ergashev.tukhtasin@gmail.com 


\section{Введение. Предварительные сведения}

Теория потенциала, имеющая многочисленные приложения в механике жидкости, эластодинамике, электромагнетизме и акустике, позволяет свести краевые задачи для эллиптических уравнений к решению интегральных уравнений, а это, в свою очередь, облегчает процесс исследования краевых задач, особенно, когда рассматриваются задачи для эллиптических уравнений с сингулярными коэффициентами [1].

При решении всякой краевой задачи, в том числе при построении теории потенциала, для эллиптических уравнений важную роль играют фундаментальные решения данного уравнения. Фундаментальные решения более обобщенного эллиптического уравнения

$$
E_{(\alpha, \lambda)}^{(m, n)}(u) \equiv \sum_{i=1}^{m} u_{x_{i} x_{i}}+\sum_{k=1}^{n} \frac{2 \alpha_{k}}{x_{k}} u_{x_{k}}+\lambda u=0
$$

в области $\mathbb{R}_{m}^{n+}=\left\{\left(x_{1}, \ldots, x_{m}\right): x_{1}>0, \ldots, x_{n}>0\right\}$, являющейся $2^{n}$-ой частью евклидова пространства $\mathbb{R}_{m}$, найдены при $\lambda=0$ в [2], а при наличии параметра $\lambda$ - в [3]. Здесь $m$ - размерность $\mathbb{R}_{m}, n$ - число сингулярных коэффициентов уравнения (1) $(1 \leqslant n \leqslant m, m \geqslant 2) ; \alpha:=\left(\alpha_{1}, \ldots, \alpha_{n}\right)$ и $\lambda-$ действительные числа, причем $0<2 \alpha_{k}<1, k=1, \ldots, n$. Отметим, что многие авторы [4-10] занимались нахождением фундаментальных решений для различных частных случаев уравнения (1). Кроме того, в работе [11] исследована задача Дирихле для трехмерного уравнения эллиптико-гиперболического типа с тремя сингулярными коэффициентами $(m=n=3, \lambda=0)$, а в [12] методом оператора преобразования найдено решение задачи Дирихле для уравнения (1) в случае, когда $m=n$.

Пользуясь известными фундаментальными решениями [2], в работе [13] найдено в явном виде решение обобщенной задачи Хольмгрена для уравнения $E_{(\alpha, 0)}^{(m, n)}(u)=0$ в $2^{n}$-ой части многомерного шара, находящейся в $\mathbb{R}_{m}^{n+}$.

Несмотря на то, что в настоящее время известны фундаментальные решения даже для многомерного (более двумерного) уравнения, такого, как уравнение (1), построение теории потенциала ограничивалось лишь двумерными уравнениями с одним и двумя сингулярными коэффициентами.

В работах [14-16] построена теория потенциала для простейшего сингулярного эллиптического уравнения

$$
u_{x x}+u_{y y}+\frac{2 \alpha}{x} u_{x}=0, \quad 0<2 \alpha<1
$$

в области, ограниченной в полуплоскости $x>0$. Изложение результатов по теории потенциала для двумерного сингулярного эллиптического уравнения (2) со ссылками на оригинальную литературу содержится в монографии Смирнова [17], которая является основным трудом в этой области.

В работах [18-21] установлены некоторые свойства потенциалов двойного слоя, соответствующих четырем фундаментальным решениям обобщенного двуосесимметрического эллиптического уравнения

$$
u_{x x}+u_{y y}+\frac{2 \alpha}{x} u_{x}+\frac{2 \beta}{y} u_{y}=0, \quad 0<2 \alpha, \quad 2 \beta<1,
$$

в области, ограниченной в первой четверти плоскости $x O y$. 
Относительно мало работ посвящено случаям, когда размерность уравнения превышает два. Отметим лишь работы [10,22]. Поэтому вопрос исследования потенциалов двойного и простого слоев, хотя бы для трехмерного эллиптического уравнения с одним сингулярным коэффициентом, является актуальной задачей.

Рассмотрим уравнение

$$
E(u) \equiv u_{x x}+u_{y y}+u_{z z}+\frac{2 \alpha}{x} u_{x}=0, \quad 0<2 \alpha<1,
$$

в полупространстве $x>0$. В работе [23] доказываются теоремы, позволяющие выявить предельные свойства потенциалов двойного и простого слоев, соответствующих одному из фундаментальных решений уравнения (3), и в качестве приложения полученных результатов исследуется смешанная задача для уравнения (3) в области, ограниченной в полупространстве $x>0$.

В данной работе, пользуясь другим фундаментальным решением уравнения (3), построим теорию потенциала и применим ее к решению задачи Хольмгрена.

При необходимости будем использовать запись $\mathbb{R}_{3}^{+}=\{(x, y, z): x>0\}$ для обозначения полупространства $x>0$. Прежде чем перейти к изложению основных результатов, приведем необходимые сведения о специальных функциях.

Символ Похгаммера $(t)_{n}$ при целых $n$ определяется равенством

$$
(t)_{n}=t(t+1) \cdots(t+n-1), \quad n=1,2, \ldots ; \quad(t)_{0} \equiv 1 .
$$

Гипергеометрическая функция Гаусса определяется внутри круга $|t|<1$ как сумма гипергеометрического ряда [24, гл. 2, § 2.1, формула (2)]

$$
F(a, b ; c ; t)=\sum_{k=0}^{\infty} \frac{(a)_{k}(b)_{k}}{k !(c)_{k}} t^{k}
$$

а при $|t| \geqslant 1$ получается аналитическим продолжением этого ряда. В формуле (5) параметры $a, b, c$ и переменная $t$ могут быть комплексными, причем $c \neq 0,-1, \ldots$, a $(t)_{k}$ - символ Похгаммера $(4)$.

Одно из фундаментальных решений уравнения (3) имеет вид [2]

$$
q(x, y, z ; \xi, \eta, \zeta)=\frac{1}{2 \pi} r^{-2 \alpha-1} F\left(\alpha+\frac{1}{2}, \alpha ; 2 \alpha ; \sigma\right)
$$

где

$$
\left.0<2 \alpha<1, \quad \sigma=1-\frac{r_{1}^{2}}{r^{2}} ; \quad \begin{array}{l}
r_{1}^{2} \\
r^{2}
\end{array}\right\}=(x \pm \xi)^{2}+(y-\eta)^{2}+(z-\zeta)^{2} .
$$

Эта функция по переменным $(x, y, z)$ является решением уравнения $(3)$, имеет особенность порядка $1 / r$ при $r \rightarrow 0$ и, следовательно, действительно является фундаментальным решением уравнения (3). Нетрудно видеть, что

$$
\left.\left(x^{2 \alpha} \frac{\partial q(x, y, z ; \xi, \eta, \zeta)}{\partial x}\right)\right|_{x=0}=\left.\left(\xi^{2 \alpha} \frac{\partial q(x, y, z ; \xi, \eta, \zeta)}{\partial \xi}\right)\right|_{\xi=0}=0
$$

для всех $y, z, \eta$ и $\zeta$. 


\section{1. Формулы Грина}

Рассмотрим тождество

$$
\begin{aligned}
x^{2 \alpha}[u E(v)-v E(u)]=\frac{\partial}{\partial x}\left[x^{2 \alpha}(\right. & \left.\left.v_{x} u-v u_{x}\right)\right]+ \\
& +x^{2 \alpha} \frac{\partial}{\partial y}\left(v_{y} u-v u_{y}\right)+x^{2 \alpha} \frac{\partial}{\partial z}\left(v_{z} u-v u_{z}\right),
\end{aligned}
$$

интегрируя обе части которого по области $D$, расположенной в полупространстве $x>0$, и пользуясь формулой Гаусса-Остроградского, получим

$$
\iiint_{D} x^{2 \alpha}[u E(v)-v E(u)] d x d y d z=\iint_{S}\left(u B_{n}^{\alpha}[v]-v B_{n}^{\alpha}[u]\right) d S,
$$

где $S$ - граница области $D, n$ - внешняя нормаль к поверхности $S$ и

$$
B_{n}^{\alpha}[\cdot]=x^{2 \alpha}\left[\cos (n, x) \frac{\partial(\cdot)}{\partial x}+\cos (n, y) \frac{\partial(\cdot)}{\partial y}+\cos (n, z) \frac{\partial(\cdot)}{\partial z}\right]
$$

Здесь и далее (ради краткости записи) буква $n$ в нижнем индексе обозначения $B_{n}^{\alpha}[\cdot]$ указывает на то, что конормальная производная вычисляется по переменным $x, y$ и $z$ (сравни с определением (15) ниже).

Формула Грина (9) выводится при следующих предположениях:

- функции $u(x, y, z), v(x, y, z)$ и их частные производные первого порядка непрерывны в замкнутой области $\bar{D}$,

- частные производные второго порядка непрерывны внутри $D$ и интегралы по $D$, содержащие $E(u)$ и $E(v)$, имеют смысл.

Если $E(u)$ и $E(v)$ не обладают непрерывностью вплоть до $S$, то этонесобственные интегралы, которые получаются как пределы по любой последовательности областей $D_{k}$, которые содержатся внутри $D$, когда эти области $D_{k}$ стремятся к $D$, так что всякая точка, находящаяся внутри $D$ попадает внутрь областей $D_{k}$, начиная с некоторого номера $k$.

Если $u$ и $v$-решения уравнения (3), то из формулы (9) имеем

$$
\iint_{S}\left(u B_{n}^{\alpha}[v]-v B_{n}^{\alpha}[u]\right) d S=0
$$

Полагая в формуле $(9) v \equiv 1$ и заменяя $u$ на $u^{2}$, получим

$$
\iiint_{D} x^{2 \alpha}\left[\left(\frac{\partial u}{\partial x}\right)^{2}+\left(\frac{\partial u}{\partial y}\right)^{2}+\left(\frac{\partial u}{\partial z}\right)^{2}\right] d x d y d z=\iint_{S} u B_{n}^{\alpha}[u] d S
$$

где $u(x, y, z)$ - решение уравнения $(3)$.

Наконец, из формулы (11), полагая $v \equiv 1$, будем иметь

$$
\iint_{S} B_{n}^{\alpha}[u] d S=0
$$

т.е. интеграл от конормальной производной решения уравнения (3) по замкнутой поверхности $S$ области $D$ равен нулю. 


\section{2. Потенциал двойного слоя}

Пусть $\Gamma$ - поверхность Ляпунова, лежащая в полупространстве $x>0$, и пусть $D$ - область, ограниченная односвязной открытой областью $X$ плоскости $y O z$ и поверхностью Г. Общую границу плоской области $X$ и поверхности Г обозначим через $\gamma$.

Параметрическое уравнение поверхности $\Gamma$ пусть будет $x=x(s, t), y=$ $=y(s, t), z=z(s, t),(s, t) \in \bar{\Phi}$, где $\Phi:=\left(s_{1}, s_{2}\right) \times\left(t_{1}, t_{2}\right)$ - область изменения $s$ и $t$. Тогда параметрическое уравнение плоской кривой $\gamma$ будет иметь вид $y=y\left(s, t_{0}\right), z=z\left(s, t_{0}\right)$, где $t_{0} \in\left[t_{1}, t_{2}\right]$ - фиксированное число, удовлетворяющее уравнению $x\left(s, t_{0}\right)=0$ при любых значениях $s \in\left[s_{1}, s_{2}\right]$. Относительно поверхности Г будем предполагать, что:

1) функции $x(s, t), y(s, t)$ и $z(s, t)$ имеют непрерывные частные производные первого порядка по $s$ и $t$ в $\bar{\Phi}$, не обращающиеся одновременно в нуль $\left(x_{s}^{2}+y_{s}^{2}+z_{s}^{2} \neq 0, x_{t}^{2}+y_{t}^{2}+z_{t}^{2} \neq 0\right)$

2) при стремлении точек поверхности $\Gamma$ к точкам кривой $\gamma$ поверхность $\Gamma$ образует прямой угол с плоскостью $x=0$.

Координаты переменной точки на поверхности Г будем обозначать через $(\xi, \eta, \zeta)$, где $\xi=\xi(\theta, \vartheta), \eta=\eta(\theta, \vartheta), \zeta=\zeta(\theta, \vartheta),(\theta, \vartheta) \in \bar{\Phi}$.

Рассмотрим интеграл

$$
w(x, y, z)=\iint_{\Gamma} \mu(\theta, \vartheta) B_{\nu}^{\alpha}[q(\xi, \eta, \zeta ; x, y, z)] d \theta d \vartheta
$$

где $\mu(\theta, \vartheta) \in C(\bar{\Gamma})$ и $q(\xi, \eta, \zeta ; x, y, z)$ - фундаментальное решение уравнения (3), определенное формулой (6),

$$
B_{\nu}^{\alpha}[\cdot]=\xi^{2 \alpha}\left[\cos (\nu, \xi) \frac{\partial(\cdot)}{\partial \xi}+\cos (\nu, \eta) \frac{\partial(\cdot)}{\partial \eta}+\cos (\nu, \zeta) \frac{\partial(\cdot)}{\partial \zeta}\right],
$$

$\nu$ - внешняя нормаль к поверхности Г. Здесь и далее (опять ради краткости записи) буква $\nu$ в нижнем индексе обозначения $B_{\nu}^{\alpha}[\cdot]$ указывает на то, что конормальная производная вычисляется по переменным $\xi, \eta$ и $\zeta$ (сравни с определением (10)).

ОПРедЕЛЕНиЕ 1. Интеграл (14) будем называть потенииалом двойного слоя с плотностъю $\mu(\theta, \vartheta)$.

Очевидно, что $w(x, y, z)$ есть регулярное решение уравнения (3) в любой области, лежащей в полупространстве $x>0$, не имеющей общих точек ни с поверхностью $\Gamma$, ни с плоскостью $y O z$. Как и в случае логарифмического потенциала, можно показать существование потенциала двойного слоя (14) в точках поверхности Г для ограниченной плотности $\mu(\theta, \vartheta)$.

Лемма 1. Справедлива следующая формула:

$$
w_{1}(x, y, z) \equiv \iint_{\Gamma} B_{\nu}^{\alpha}[q(\xi, \eta, \zeta ; x, y, z)] d \Gamma=\left\{\begin{array}{rlrl}
-1, & & (x, y, z) & \in D \cup X \\
-1 / 2, & & (x, y, z) \in \Gamma \cup \gamma, \\
0, & & (x, y, z) \notin \bar{D} .
\end{array}\right.
$$

До ка з а т ель с т в о. Процесс доказательства состоит из нескольких случаев. 
1. Пусть точка $(x, y, z)$ лежит вне области $D$ и над плоскостью $y O z$. Тогда $q(\xi, \eta, \zeta ; x, y, z)$ есть регулярное решение уравнения (3) внутри области $D$ с непрерывными производными всех порядков вплоть до поверхности Г, и в силу (13)

$$
w_{1}(x, y, z) \equiv \iint_{\Gamma} B_{\nu}^{\alpha}[q(\xi, \eta, \zeta ; x, y, z)] d \theta d \vartheta=0 .
$$

2. Пусть точка $(x, y, z)$ находится внутри $D$. Вырежем из области $D$ шар малого радиуса $\rho$ с центром в точке $(x, y, z)$ и обозначим через $D_{\rho}$ оставшуюся часть области $D$, а через $C_{\rho}$ - сферу вырезанного шара. В области $D_{\rho}$ функция $q(\xi, \eta, \zeta ; x, y, z)$ - регулярное решение уравнения (3) и, согласно (7) и $(13)$, мы имеем

$$
\iint_{\Gamma} B_{\nu}^{\alpha}[q(\xi, \eta, \zeta ; x, y, z)] d \theta d \vartheta+\iint_{C_{\rho}} B_{\nu}^{\alpha}[q(\xi, \eta, \zeta ; x, y, z)] d C_{\rho}=0
$$

т.e.

$$
w_{1}(x, y, z)=-\iint_{C_{\rho}} B_{\nu}^{\alpha}[q(\xi, \eta, \zeta ; x, y, z)] d C_{\rho} .
$$

Вычислим производную по нормали $\nu$ от фундаментального решения $q(\xi, \eta, \zeta ; x, y, z)$. Применяя последовательно формулу для вычисления производной гипергеометрической функции Гаусса $[24$, гл. $2, \S 2.8$, формула (20)]

$$
\frac{d}{d \sigma} F(a, b ; c ; \sigma)=\frac{a b}{c} F(a+1, b+1 ; c+1 ; \sigma)
$$

и смежное соотношение

$$
\frac{b}{c} \sigma F(a+1, b+1 ; c+1 ; \sigma)=F(a+1, b ; c ; \sigma)-F(a, b ; c ; \sigma),
$$

получим

$$
\begin{aligned}
\frac{\partial q(\xi, \eta, \zeta ; x, y, z)}{\partial \xi}=\frac{1+2 \alpha}{2 \pi} & (x-\xi) r^{-2 \alpha-3} F\left(\alpha+\frac{3}{2}, \alpha ; 2 \alpha ; \sigma\right)- \\
& -\frac{1+2 \alpha}{2 \pi} x r^{-2 \alpha-3} F\left(\alpha+\frac{3}{2}, 1+\alpha ; 1+2 \alpha ; \sigma\right) .
\end{aligned}
$$

В результате применения формулы дифференцирования [24, гл. $2, \S 2.8$, формула (21)]

$$
\frac{d}{d \sigma}\left[\sigma^{a} F(a, b ; c ; \sigma)\right]=a \sigma^{a-1} F(a+1, b ; c ; \sigma)
$$

находим

$$
\begin{aligned}
& \frac{\partial q(\xi, \eta, \zeta ; x, y, z)}{\partial \eta}=\frac{1+2 \alpha}{2 \pi}(y-\eta) r^{-2 \alpha-3} F\left(\alpha+\frac{3}{2}, \alpha ; 2 \alpha ; \sigma\right) \\
& \frac{\partial q(\xi, \eta, \zeta ; x, y, z)}{\partial \zeta}=\frac{1+2 \alpha}{2 \pi}(z-\zeta) r^{-2 \alpha-3} F\left(\alpha+\frac{3}{2}, \alpha ; 2 \alpha ; \sigma\right)
\end{aligned}
$$


Используя (18), (19) и (20), с учетом (15) имеем

$$
\begin{aligned}
B_{\nu}^{\alpha}[q(\xi, \eta, \zeta ; x, y, z)] & =\frac{1+2 \alpha}{2 \pi} r^{-2 \alpha-1} F\left(\alpha+\frac{3}{2}, \alpha ; 2 \alpha ; \sigma\right) B_{\nu}^{\alpha}\left[\ln \frac{1}{r}\right]- \\
& -\frac{1+2 \alpha}{2 \pi} x r^{-2 \alpha-3} \xi^{2 \alpha} F\left(\alpha+\frac{3}{2}, 1+\alpha ; 1+2 \alpha ; \sigma\right) \cos (\nu, \xi) .
\end{aligned}
$$

Далее, применив известную формулу [24, гл. $2, \S 2.9$, формула (2)]

$$
F(a, b ; c ; x)=(1-x)^{-b} F\left(c-a, b ; c ; \frac{x}{x-1}\right)
$$

к каждой гипергеометрической функции в (21), получим

$$
\begin{aligned}
B_{\nu}^{\alpha}[q(\xi, \eta, \zeta ; x, y, z)] & =-\frac{1+2 \alpha}{4 \pi r_{1}^{2 \alpha} r} F\left(\alpha-\frac{3}{2}, \alpha ; 2 \alpha ; 1-\frac{r^{2}}{r_{1}^{2}}\right) B_{\nu}^{\alpha}\left[\ln r^{2}\right]- \\
& -\frac{1+2 \alpha}{2 \pi r r_{1}^{2 \alpha+2}} x \xi^{2 \alpha} F\left(\alpha-\frac{1}{2}, 1+\alpha ; 1+2 \alpha ; 1-\frac{r^{2}}{r_{1}^{2}}\right) \cos (\nu, \xi) .
\end{aligned}
$$

Теперь равенство (17) может быть написано так:

$$
w_{1}(x, y, z)=i_{1}(x, y, z)+j_{1}(x, y, z)
$$

где

$$
\begin{gathered}
i_{1}(x, y, z)=-\frac{1+2 \alpha}{2 \pi} \iint_{C_{\rho}} \frac{1}{r_{1}^{2 \alpha} r} F\left(\alpha-\frac{3}{2}, \alpha ; 2 \alpha ; 1-\frac{r^{2}}{r_{1}^{2}}\right) B_{\nu}^{\alpha}\left[\ln \frac{1}{r}\right] d C_{\rho}, \\
j_{1}(x, y, z)=\frac{1+2 \alpha}{2 \pi} x \iint_{C_{\rho}} \frac{\xi^{2 \alpha}}{r_{1}^{2 \alpha} r} F\left(\alpha-\frac{1}{2}, 1+\alpha ; 1+2 \alpha ; 1-\frac{r^{2}}{r_{1}^{2}}\right) \cos (\nu, \xi) d C_{\rho} .
\end{gathered}
$$

Преобразуем правую часть равенства (24). На сфере нормаль направлена против радиуса. Отсюда

$$
i_{1}(x, y, z)=-\frac{1+2 \alpha}{2 \pi} \iint_{C_{\rho}} \frac{\xi^{2 \alpha}}{r_{1}^{2 \alpha} r^{2}} F\left(\alpha-\frac{3}{2}, \alpha ; 2 \alpha ; 1-\frac{r^{2}}{r_{1}^{2}}\right) d C_{\rho} .
$$

Вводя сферические координаты

$$
\begin{gathered}
\xi=x+\rho \cos \varphi, \quad \eta=y+\rho \sin \varphi \cos \psi, \quad \zeta=z+\rho \sin \varphi \sin \psi \\
(\rho \geqslant 0, \quad 0 \leqslant \varphi \leqslant \pi, \quad 0 \leqslant \psi \leqslant 2 \pi)
\end{gathered}
$$

в интеграле (25), получим

$$
\begin{aligned}
i_{1}(x, y, z)=-\frac{1+2 \alpha}{2 \pi} \int_{0}^{2 \pi} d \psi \int_{0}^{\pi}\left[\frac{x^{2}+2 x \rho \cos \varphi+\rho^{2} \cos ^{2} \varphi}{4 x^{2}+4 x \rho \cos \varphi+\rho^{2}}\right]^{\alpha} \times \\
\quad \times F\left(\alpha-\frac{3}{2}, \alpha ; 2 \alpha ; \frac{4 x^{2}+4 x \rho \cos \varphi}{4 x^{2}+4 x \rho \cos \varphi+\rho^{2}}\right) \sin \varphi d \varphi .
\end{aligned}
$$


Теперь в правой части равенства (27) переходим к пределу при $\rho \rightarrow 0$. Используя формулу суммирования для гипергеометрической функции Гаусса $[24$, гл. $2, \S 2.8$, формула (46)]

$$
F(a, b ; c ; 1)=\frac{\Gamma(c) \Gamma(c-a-b)}{\Gamma(c-a) \Gamma(c-b)}, \quad \operatorname{Re}(c-a-b)>0 ; \quad c \neq 0,-1,-2, \ldots,
$$

получим

$$
\lim _{\rho \rightarrow 0} i_{1}(x, y, z)=-1
$$

Еще проще доказывается, что

$$
\lim _{\rho \rightarrow 0} j_{1}(x, y, z)=0 .
$$

Подставляя теперь (28) и (29) в (23), получим

$$
w_{1}(x, y, z)=-1, \quad(x, y, z) \in D .
$$

3. Пусть теперь точка $(x, y, z)$ совпадает с некоторой точкой $M_{0}\left(x_{0}, y_{0}, z_{0}\right)$, лежащей на поверхности $Г$. Проведем сферу малого радиуса $\rho$ с центром в точке $M_{0}$. Эта сфера вырежет часть $\Gamma_{\rho}$ поверхности $Г$. Оставшуюся часть поверхности обозначим через $\Gamma_{\rho}^{*}$. Мы имеем

$$
w_{1}\left(x_{0}, y_{0}, z_{0}\right)=\lim _{\rho \rightarrow 0} \iint_{\Gamma_{\rho}^{*}} B_{\nu}^{\alpha}\left[q\left(\xi, \eta, \zeta ; x_{0}, y_{0}, z_{0}\right)\right] d \Gamma_{\rho}^{*} .
$$

Обозначим через $C_{\rho}^{*}$ часть сферы $C_{\rho}$, лежащей внутри области $D$, и рассмотрим область, ограниченную поверхностями $\Gamma_{\rho}^{*}, C_{\rho}^{*}$ и плоской областью $X$ плоскости $y O z$. Так как точка $M_{0}$ лежит вне этой области, в этой области $q(\xi, \eta, \zeta ; x, y, z)$ - регулярное решение уравнения (3), и в силу (13) имеем

$$
\iint_{\Gamma_{\rho}^{*}} B_{\nu}^{\alpha}\left[q\left(\xi, \eta, \zeta ; x_{0}, y_{0}, z_{0}\right)\right] d \Gamma_{\rho}^{*}=\iint_{C_{\rho}^{*}} B_{\nu}^{\alpha}\left[q\left(\xi, \eta, \zeta ; x_{0}, y_{0}, z_{0}\right)\right] d C_{\rho}^{*} .
$$

Подставляя (31) в (30), получим

$$
w_{1}\left(x_{0}, y_{0}, z_{0}\right)=-\lim _{\rho \rightarrow 0} \iint_{C_{\rho}^{*}} B_{\nu}^{\alpha}\left[q\left(\xi, \eta, \zeta ; x_{0}, y_{0}, z_{0}\right)\right] d C_{\rho}^{*}
$$

Вводя снова сферические координаты (26) с центром в точке $M_{0}$, получим

$$
w_{1}(x, y, z)=-1 / 2, \quad(x, y, z) \in \Gamma .
$$

4. Положим, наконец, что точка $(x, y, z)$ находится на плоскости $y O z$. Проведем плоскость $x=\delta$ ( $\delta>0$ достаточно мало) и рассмотрим область $D_{\delta}$, которая есть часть области $D$, лежащая над плоскостью $x=\delta$. Применяя формулу (13), получим 


$$
\begin{aligned}
w_{1}(0, y, z)=\iint_{H_{\delta}} B_{\nu}^{\alpha}[q(\xi, \eta, \zeta ; 0, y, z)] & d H_{\delta}+ \\
& +\iint_{X_{\delta}}\left[\xi^{2 \alpha} \frac{\partial q(\xi, \eta, \zeta ; 0, y, z)}{\partial \xi}\right]_{\xi=\delta} d X_{\delta},
\end{aligned}
$$

где $H_{\delta}$ - часть поверхности $\Gamma$, находящаяся ниже плоскости $x=\delta$, a $X_{\delta}-$ сечение области $D$ плоскостью $x=\delta$, т.е. $X_{\delta}$ - плоская область, ограниченная замкнутой кривой $\gamma_{\delta}: y=y\left(s, t_{\delta}\right), z=z\left(s, t_{\delta}\right)$, здесь $t_{\delta} \in\left[t_{1}, t_{2}\right]$ определяется из уравнения $x\left(s, t_{\delta}\right)=\delta$ при любых значениях $s \in\left[s_{1}, s_{2}\right]$.

Нетрудно видеть, что интеграл

$$
\iint_{H_{\delta}} B_{\nu}^{\alpha}[q(\xi, \eta, \zeta ; 0, y, z)] d H_{\delta}
$$

при $\delta \rightarrow 0$ стремится к нулю при любом значении $y$ и $z$.

Пусть $X_{\delta}=\{(y, z): p(\delta)<y<q(\delta), h(y, \delta)<z<k(y, \delta)\}$, где $p(\delta)$ и $q(\delta)$ некоторые конечные действительные числа, зависящие от $\delta$, а $z=h(y, \delta)$, $z=k(y, \delta)$ - непрерывные функции на отрезке $[p(\delta), q(\delta)]$. Далее, согласно (18), выражение (32) можно записать в виде

$$
\begin{aligned}
& w_{1}(0, y, z)= \\
& =-\frac{1+2 \alpha}{2 \pi} \lim _{\delta \rightarrow 0} \delta^{1+2 \alpha} \int_{p(\delta)}^{q(\delta)} \int_{h(\zeta, \delta)}^{k(\zeta, \delta)}\left[\delta^{2}+(\eta-y)^{2}+(\zeta-z)^{2}\right]^{-\alpha-3 / 2} d \eta d \zeta .
\end{aligned}
$$

Преобразуем выражение (33). Вместо $\eta$ и $\zeta$ введем новые переменные интегрирования $t=(\eta-y) / \delta$ и $s=(\zeta-z) / \delta$. Совершая замену переменных, получим

$$
w_{1}(0, y, z)=-\frac{1+2 \alpha}{2 \pi} \lim _{\delta \rightarrow 0} \int_{\alpha_{1}}^{\alpha_{2}} \int_{\beta_{1}}^{\beta_{2}}\left(1+t^{2}+s^{2}\right)^{-\alpha-3 / 2} d t d s
$$

где

$\alpha_{1}=\frac{p(\delta)-y}{\delta}, \quad \alpha_{2}=\frac{q(\delta)-y}{\delta}, \quad \beta_{1}=\frac{h(\eta+t \delta, \delta)-z}{\delta}, \quad \beta_{2}=\frac{k(\eta+t \delta, \delta)-z}{\delta}$.

Известно [25, гл. 4, § 4.6, формула 4.638.3], что

$$
\int_{-\infty}^{\infty} \int_{-\infty}^{\infty}\left(1+t^{2}+s^{2}\right)^{-\alpha-3 / 2} d t d s=\frac{2 \pi}{1+2 \alpha}
$$

Таким образом, из формулы (34) в силу условий 1) и 2), наложенных на поверхность Г, и формулы (35) будем иметь

$$
w_{1}(0, y, z)=\left\{\begin{aligned}
-1, & (y, z) \in X \\
-1 / 2, & (y, z) \in \gamma \\
0, & (y, z) \notin X \cup \gamma
\end{aligned}\right.
$$

Лемма 1 доказана. 
ЛЕмма 2. Если поверхность Г удовлетворяет перечисленным выше условиям, то

$$
\iint_{\Gamma}\left|B_{\nu}^{\alpha}[q(\xi, \eta, \zeta ; x, y, z)]\right| d \theta d \vartheta \leqslant B
$$

где $B-$ постоянная.

До ка з а тель с т в о. Проведем плоскость $x=\delta(\delta>0$ достаточно мало) и части поверхности $\Gamma$, находящиеся ниже и выше этой плоскости, обозначим через $H_{\delta}$ и $L_{\delta}$, соответственно. Формулу (22) представим в виде

$$
B_{\nu}^{\alpha}[q(\xi, \eta, \zeta ; x, y, z)]=P(\xi, \eta, \zeta ; x, y, z)+Q(\xi, \eta, \zeta ; x, y, z),
$$

где

$$
\begin{gathered}
P(\xi, \eta, \zeta ; x, y, z)=-\frac{1+2 \alpha}{2 \pi r r_{1}^{2+2 \alpha}} x \xi^{2 \alpha} F\left(\alpha-\frac{1}{2}, 1+\alpha ; 1+2 \alpha ; 1-\frac{r^{2}}{r_{1}^{2}}\right) \cos (\nu, \xi), \\
Q(\xi, \eta, \zeta ; x, y, z)=-\frac{1+2 \alpha}{4 \pi r r_{1}^{2 \alpha}} F\left(\alpha-\frac{3}{2}, \alpha ; 2 \alpha ; 1-\frac{r^{2}}{r_{1}^{2}}\right) B_{\nu}^{\alpha}\left[\ln r^{2}\right] .
\end{gathered}
$$

Из теории специальных функций известно, что гипергеометрическая функция Гаусса $F(a, b ; c ; t)$ при $\operatorname{Re}(c-a-b)>0$ и $|t| \leqslant 1$ ограничена. Кроме того, согласно теории потенциала для уравнения Лапласа имеет место неравенство

$$
\left|\iint_{\Gamma} \mu \frac{\cos (r, \nu)}{r^{2}} d \theta d \vartheta\right|<C
$$

где $\mu$ - ограниченная интегрируемая функция, а $C$ - некоторая постоянная.

Теперь легко видеть, что

$$
\iint_{L_{\delta}}|P(\xi, \eta, \zeta ; x, y, z)| d L_{\delta} \leqslant C_{1}, \quad \delta>0,
$$

где $C_{1}$ не зависит от $x, y$ и $z$.

В силу неравенства $r_{1}^{2}>r^{2}$ имеем

$$
\iint_{H_{\delta}}|P(\xi, \eta, \zeta ; x, y, z)| d H_{\delta} \leqslant C_{2} \iint_{H_{\delta}} \frac{|\cos (\nu, \xi)|}{r^{2}} d H_{\delta} \leqslant C_{3} .
$$

Далее, проводя аналогичные рассуждения, получим

$$
\iint_{\Gamma}|Q| d \Gamma \leqslant C_{4} \iint_{\Gamma} \frac{1}{r r_{1}^{2 \alpha}}\left|B_{\nu}^{\alpha}[\ln r]\right| d \theta d \vartheta \leqslant C_{5} \iint_{\Gamma} \frac{|\cos (\nu, \xi)|}{r^{2}} d \theta d \vartheta \leqslant C_{6} .
$$

Таким образом, из полученных оценок (36), (37), (38) следует справедливость леммы 2.

Лемма 3. Если точка $(x, y, z)$ лежит на $\Gamma$, то

$$
\left|B_{\nu}^{\alpha}[q(\xi, \eta, \zeta ; x, y, z)]\right| \leqslant \frac{B_{1}}{r_{1}^{2 \alpha} r}
$$

где $B_{1}-$ постоянная. 
До ка з а т е ль с т в о. Оценка непосредственно следует из формулы (22).

Формулы (16) показывают, что при $\mu \equiv 1$ потенциал двойного слоя испытывает разрыв непрерывности, когда точка $(x, y, z)$ пересекает поверхность $Г$. В случае произвольной непрерывной плотности $\mu(s, t)$ имеет место

Теорема 1. Потенииал двойного слоя $w(x, y, z)$ имеет пределъ при стремлении точки $(x, y, z)$ к точке $\left(x_{0}, y_{0}, z_{0}\right)$ поверхности Г извне или изнутри. Если предел значений $w(x, y, z)$ изнутри обозначить через $w_{i}(s, t)$, а предел извне - через $w_{e}(s, t)$, то имеют место формуль

$$
\begin{aligned}
& w_{i}(s, t)=-\frac{1}{2} \mu(s, t)+\iint_{\Gamma} \mu(\theta, \vartheta) K(s, t ; \theta, \vartheta) d \theta d \vartheta \\
& w_{e}(s, t)=\frac{1}{2} \mu(s, t)+\iint_{\Gamma} \mu(\theta, \vartheta) K(s, t ; \theta, \vartheta) d \theta d \vartheta
\end{aligned}
$$

¿əe

$$
K(s, t ; \theta, \vartheta)=B_{\nu}^{\alpha}[q(\xi(\theta, \vartheta), \eta(\theta, \vartheta), \zeta(\theta, \vartheta) ; x(s, t), y(s, t), z(s, t))]
$$

Д о каз а те ль с т в о. Доказательство теоремы 1 следует из лемм 1 и 2.

Функция

$$
w_{0}(s, t)=\iint_{\Gamma} \mu(\theta, \vartheta) K(s, t ; \theta, \vartheta) d \theta d \vartheta
$$

непрерывна при $(x, y, z) \in \bar{\Gamma}$, что следует из хода доказательства теоремы 1. Принимая во внимание формулы $(40)$ и непрерывность функций $w_{0}(s, t)$ и $\mu(s, t)$ при $(s, t) \in\left[s_{1}, s_{2}\right] \times\left[t_{1}, t_{2}\right]$, можно утверждать, что потенциал двойного слоя $w(x, y, z)$ есть функция, непрерывная внутри области $D$ вплоть до поверхности $\Gamma$. Точно так же $w(x, y, z)$ непрерывна вне области $D$ вплоть до поверхности Г.

\section{3. Потенциал простого слоя}

Пусть поверхность Г удовлетворяет условиям 1) и 2) п. 2.

ОПРЕДЕЛЕНИЕ 2. Потеницалом простого слоя с плотностью $\rho(\theta, \vartheta)$ назовем функцию

$$
v(x, y, z)=\iint_{\Gamma} \rho(\theta, \vartheta) q(\xi, \eta, \zeta ; x, y, z) d \theta d \vartheta
$$

где $q(\xi, \eta, \zeta ; x, y, z)$ - фундаментальное решение уравнения (3).

Будем предполагать, что $\rho(\theta, \vartheta)$ - непрерывная функция на $\bar{\Gamma}$. Потенциал простого слоя (41) определен во всем полупространстве $x>0$ и остается непрерывным при переходе через поверхность Г. Очевидно, что потенциал простого слоя $v(x, y, z)$ есть регулярное решение уравнения (3) в любой области, лежащей в полупространстве $x>0$, не имеющей общих точек ни с поверхностью $\Gamma$, ни с плоскостью $y O z$. Нетрудно видеть, что при стремлении точки $(x, y, z)$ к бесконечности потенциал простого слоя $v(x, y, z)$ стремится к нулю. Действительно, пусть точка $(x, y, z)$ находится на полусфере $C_{R}$ : $x^{2}+y^{2}+z^{2}=R^{2}, x>0$, тогда в силу (6) имеем 


$$
|v(x, y, z)| \leqslant \iint_{\Gamma}|\rho(\theta, \vartheta)||q(\xi, \eta, \zeta ; x, y, z)| d \Gamma \leqslant M R^{-1-2 \alpha}, \quad R \geqslant R_{0}
$$

где $M-$ постоянная.

3.1. Конормальная производная потенциала простого слоя. Возьмем на поверхности Г произвольную точку $N(x(s, t), y(s, t), z(s, t))$ и проведем в этой точке нормаль. Рассмотрим на этой нормали какую-нибудь точку $M(x, y, z)$, не лежащую на поверхности $\Gamma$, и составим конормальную производную от потенциала простого слоя (41):

$$
B_{n}^{\alpha}[v(x, y, z)]=\iint_{\Gamma} \rho(\theta, \vartheta) B_{n}^{\alpha}[q(\xi, \eta, \zeta ; x, y, z)] d \theta d \vartheta
$$

где $B_{n}^{\alpha}[\cdot]$ - конормальная производная по переменным $x, y$ и $z$, определенная формулой (10).

Интеграл (43) существуют и в том случае, когда точка $M(x, y, z)$ совпадает с точкой $N\left(x_{0}, y_{0}, z_{0}\right)$, упомянутой выше.

Обозначим через $B_{n}^{\alpha}[v(x, y, z)]_{i}$ и $B_{n}^{\alpha}[v(x, y, z)]_{e}$ соответственно предельные значения конормальной производной при стремлении точки $M(x, y, z)$ к точке $N \in \Gamma$ изнутри и извне поверхности $\Gamma$.

Теорема 2. Для непрерывной плотности $\rho(\xi, \eta, \zeta)$ имеют место следующие формулы:

$$
\begin{aligned}
& B_{n}^{\alpha}[v(x, y, z)]_{i}=\frac{1}{2} \rho(s, t)+\iint_{\Gamma} \rho(\theta, \vartheta) K(\theta, \vartheta ; s, t) d \theta d \vartheta \\
& B_{n}^{\alpha}[v(x, y, z)]_{e}=-\frac{1}{2} \rho(s, t)+\iint_{\Gamma} \rho(\theta, \vartheta) K(\theta, \vartheta ; s, t) d \theta d \vartheta
\end{aligned}
$$

əде

$$
K(\theta, \vartheta ; s, t)=B_{n}^{\alpha}[q(\xi(\theta, \vartheta), \eta(\theta, \vartheta), \zeta(\theta, \vartheta) ; x(s, t), y(s, t), z(s, t))]
$$

Из этих формул непосредственно следует величина скачка конормальной производной потенциала простого слоя:

$$
B_{n}^{\alpha}[v(x, y, z)]_{i}-B_{n}^{\alpha}[v(x, y, z)]_{e}=\rho(x, y, z) .
$$

Точно так же, как и в неравенстве (42), можно показать, что имеет место следующая оценка:

$$
\left|B_{n}^{\alpha}[v(x, y, z)]\right| \leqslant M R^{-2-2 \alpha}, \quad R \geqslant R_{0},
$$

где $M-$ постоянная.

3.2. Применимость формулы Грина для потенциалов. Покажем, что для потенциала простого слоя (41) применима формула Грина (12). Рассмотрим область $D_{\varepsilon, \delta}$, лежащую внутри $D$ и ограниченную поверхностью $\Gamma_{\varepsilon}$, параллельной поверхности $\Gamma$, и замкнутой областью $X_{\delta}$, т.е. сечением области $D_{\varepsilon, \delta}$ плоскостью $x=\delta$. Применим формулу Грина (12) к потенциалу простого 
слоя $v(x, y, z)$, выбирая за область интегрирования область $D_{\varepsilon, \delta}$. Тогда получим

$$
\begin{aligned}
& \iiint_{D_{\varepsilon, \delta}} x^{2 \alpha}\left[\left(\frac{\partial v}{\partial x}\right)^{2}+\left(\frac{\partial v}{\partial y}\right)^{2}+\left(\frac{\partial v}{\partial z}\right)^{2}\right] d x d y d z= \\
& \quad=\iint_{\Gamma_{\varepsilon}} v B_{n}^{\alpha}[v] d \Gamma_{\varepsilon}+\iint_{X_{\delta}} \delta^{2 \alpha} v(\delta, y, z) \frac{\partial v(\delta, y, z)}{\partial x} d y d z .
\end{aligned}
$$

Конормальная производная $B_{n}^{\alpha}[v(x, y, z)]$ есть непрерывная функция вплоть до поверхности Ги

$$
\lim _{x \rightarrow 0} x^{2 \alpha} \frac{\partial v(x, y, z)}{\partial x}=0, \quad(y, z) \in X
$$

Следовательно,

$$
\lim _{\delta \rightarrow 0} \iint_{X_{\delta}} \delta^{2 \alpha} v(\delta, y, z) \frac{\partial v(\delta, y, z)}{\partial x} d y d z=0 .
$$

Переходя в формуле (47) к пределу при $\varepsilon \rightarrow 0, \delta \rightarrow 0$ и принимая во внимание (48), получим

$$
\iiint_{D} x^{2 \alpha}\left[\left(\frac{\partial v}{\partial x}\right)^{2}+\left(\frac{\partial v}{\partial y}\right)^{2}+\left(\frac{\partial v}{\partial z}\right)^{2}\right] d x d y d z=\iint_{\Gamma} v B_{n}^{\alpha}[v(x, y, z)]_{i} d \Gamma .
$$

Плоскую область, ограниченную кривой $\gamma$ и окружностью $y^{2}+z^{2}=R^{2}$, лежащей в плоскости $y O z$, обозначим через $X_{0 R}$. Применим теперь формулу (49) к области $D_{R}^{\prime}$, ограниченной поверхностью $\Gamma$, областью $X_{0 R}$ и полусферой $C_{R}$, содержащей область $D$. Переходя затем к пределу при $R \rightarrow \infty$ и учитывая (42) и (46), получим

$$
\iiint_{D^{\prime}} x^{2 \alpha}\left[\left(\frac{\partial v}{\partial x}\right)^{2}+\left(\frac{\partial v}{\partial y}\right)^{2}+\left(\frac{\partial v}{\partial z}\right)^{2}\right] d x d y d z=-\iint_{\Gamma} v B_{n}^{\alpha}[v(x, y, z)]_{e} d \Gamma .
$$

Здесь и далее $D^{\prime}=\mathbb{R}_{3}^{+} \backslash \bar{D}$ - неограниченная область при $x>0$.

\section{4. Интегральные уравнения для плотностей}

Формулы (40) и (44) могут быть написаны как интегральные уравнения для плотностей:

$$
\begin{aligned}
& \mu(s, t)-\lambda \iint_{\Gamma} K(s, t ; \theta, \vartheta) \mu(\theta, \vartheta) d \theta d \vartheta=f(s, t), \\
& \rho(s, t)-\lambda \iint_{\Gamma} K(\theta, \vartheta ; s, t) \rho(\theta, \vartheta) d \theta d \vartheta=g(s, t),
\end{aligned}
$$

где

$$
\begin{array}{lll}
\lambda=2, & f(s, t)=-2 w_{i}(s, t), & g(s, t)=-2 B_{n}^{\alpha}[v]_{e} \\
\lambda=-2, & f(s, t)=2 w_{e}(s, t), & g(s, t)=2 B_{n}^{\alpha}[v]_{i}
\end{array}
$$


Уравнения (51) и (52) - сопряженные, и в силу леммы 3 к ним применима теория Фредгольма.

Покажем, что $\lambda=2$ не является собственным значением ядра $K(s, t ; \theta, \vartheta)$. Это утверждение эквивалентно тому, что однородное интегральное уравнение

$$
\rho(s, t)-2 \iint_{\Gamma} K(\theta, \vartheta ; s, t) \rho(\theta, \vartheta) d \theta d \vartheta=0
$$

не имеет нетривиальных решений. Пусть $\rho_{0}(\theta, \vartheta)$ - непрерывное нетривиальное решение уравнения (53). Потенциал простого слоя с плотностью $\rho_{0}(\theta, \vartheta)$ дает функцию $v_{0}(x, y, z)$, которая является решением уравнения (3) в областях $D$ и $D^{\prime}$ и у которой предельные значения конормальной производной $B_{n}^{\alpha}\left[v_{0}\right]_{e}$ равны нулю в силу уравнения (53). К потенциалу простого слоя $v_{0}(x, y, z)$ применима формула $(50)$, из которой следует, что $v_{0}(x, y, z)=\mathrm{const}$ в области $D^{\prime}$. На бесконечности потенциал простого слоя равен нулю и, следовательно, $v_{0}(x, y, z) \equiv 0$ в $D^{\prime}$, а также и на поверхности Г. Применяя теперь формулу (49), мы получим, что $v_{0}(x, y, z) \equiv 0$ и внутри области $D$. Но тогда $B_{n}^{\alpha}\left[v_{0}\right]_{i}=0$, и на основании формулы $(45)$ получим $\rho_{0}(\theta, \vartheta) \equiv 0$. Таким образом, однородное уравнение (53) имеет только тривиальное решение; следовательно, $\lambda=2$ не есть собственное значение ядра $K(s, t ; \theta, \vartheta)$.

Однородное уравнение

$$
\mu(s, t)-\lambda \iint_{\Gamma} K(s, t ; \theta, \vartheta) \mu(\theta, \vartheta) d \theta d \vartheta=0
$$

при $\lambda=-2$ имеет в силу (16) решение, равное произвольной постоянной, т.е. $\lambda=-2$ есть собственное значение ядра $K(s, t ; \theta, \vartheta)$.

\section{5. Решение задачи Хольмгрена с помощью потенциалов}

5.1. Постановка краевой задачи Хольмгрена. Пусть $D$ - область, ограниченная односвязной открытой областью $X$ плоскости $y O z$ и поверхностью Ляпунова $\Gamma$, лежащей в полупространстве $x>0$. Общую границу плоской области $X$ и поверхности Г обозначим через $\gamma$. Будем предполагать, что поверхность Г удовлетворяет условиям 1) и 2) п. 2.

5.2. Задача Хольмгрена. Найти в области $D$ регулярное решение уравнения (3), непрерывное в замкнутой области $\bar{D}$ и удовлетворяющее краевым условиям

$$
\left.u\right|_{\Gamma}=\varphi(s, t), \quad(s, t) \in \bar{\Phi} ; \quad \lim _{x \rightarrow 0} x^{2 \alpha} \frac{\partial u(x, y, z)}{\partial x}=\nu_{1}(y, z), \quad(y, z) \in X,
$$

где $\nu_{1}(y, z)$ - непрерывная функция в $X$, причем в случае, когда кривая $\gamma$ есть окружность, при стремлении точек $(y, z) \in X$ к кривой $\gamma$ функция $\nu_{1}(y, z)$ может обращаться в бесконечность порядка меньше $1-2 \alpha$.

5.3. Единственность решения задачи Хольмгрена. Рассмотрим область $D_{\varepsilon, \delta}$, лежащую внутри $D$ и ограниченную поверхностью $\Gamma_{\varepsilon}$, параллельной поверхности $\Gamma$, и замкнутой областью $X_{\delta}$, т.е. сечением области $D_{\varepsilon, \delta}$ плоскостью $x=\delta$. Интегрируя обе части тождества (8) по области $D_{\varepsilon, \delta}$ и пользуясь формулой Гаусса-Остроградского, получим 


$$
\iiint_{D_{\varepsilon, \delta}} x^{2 \alpha}[u E(v)-v E(u)] d x d y d z=\iint_{S_{\varepsilon, \delta}}\left(u B_{n}^{\alpha}[v]-v B_{n}^{\alpha}[u]\right) d S_{\varepsilon, \delta},
$$

где $S_{\varepsilon, \delta}$ - граница области $D_{\varepsilon, \delta}$.

Нетрудно убедиться в справедливости следующего равенства:

$$
\begin{aligned}
& \iiint_{D_{\varepsilon, \delta}} x^{2 \alpha} u E(u) d x d y d z=\iiint_{D_{\varepsilon, \delta}} x^{2 \alpha}\left[u_{x}^{2}+u_{y}^{2}+u_{z}^{2}\right] d x d y d z- \\
&-\iiint_{D_{\varepsilon, \delta}}\left[\frac{\partial}{\partial x}\left(x^{2 \alpha} u u_{x}\right)+x^{2 \alpha} \frac{\partial}{\partial y}\left(u u_{y}\right)+x^{2 \alpha} \frac{\partial}{\partial z}\left(u u_{z}\right)\right] d x d y d z .
\end{aligned}
$$

Применяя формулу Гаусса-Остроградского к этому тождеству, после перехода к пределу при $\delta \rightarrow 0$ и $\varepsilon \rightarrow 0$ имеем

$$
\begin{aligned}
\iiint_{D} x^{2 \alpha}\left[u_{x}^{2}+u_{y}^{2}\right. & \left.+u_{z}^{2}\right] d x d y d z= \\
& =\iint_{X} \tau_{1}(y, z) \nu_{1}(y, z) d y d z-\iint_{\Gamma} \varphi(x, y, z) B_{n x}^{\alpha}[u] d x d y d z .
\end{aligned}
$$

Если теперь рассмотрим однородную задачу Хольмгрена, то из (55) получим

$$
\iiint_{D} x^{2 \alpha}\left[u_{x}^{2}+u_{y}^{2}+u_{z}^{2}\right] d x d y d z=0
$$

Отсюда следует, что $u(x, y, z)=0$ в $\bar{D}$.

Справедлива следующая

Теорема 3. Если задача Хольмгрена для уравнения (3) имеет регулярное решение, то оно единственно.

5.4. Сведение задачи Хольмгрена к случаю однородных условий на плоскости вырождения. Покажем, что можно ограничиться случаем, когда $\nu_{1}(y, z) \equiv 0$.

Пусть $X=\{(y, z): p<y<q, h(y)<z<k(y)\}$, где $p, q$ - некоторые конечные действительные числа, а $z=h(y), z=k(y)$ - непрерывные функции на отрезке $[p, q]$.

Используя фундаментальное решение (6) уравнения (3), возьмем решение в виде

$$
v(x, y, z)=-\frac{1}{2 \pi} \iint_{X} \nu_{1}(\eta, \zeta)\left[x^{2}+(\eta-y)^{2}+(\zeta-z)^{2}\right]^{-1 / 2-\alpha} d \eta d \zeta
$$

Дифференцируя интеграл (56) по $x$, получим

$$
\frac{\partial v}{\partial x}=\frac{1+2 \alpha}{2 \pi} x \iint_{X} \nu_{1}(\eta, \zeta)\left[x^{2}+(\eta-y)^{2}+(\zeta-z)^{2}\right]^{-3 / 2-\alpha} d \eta d \zeta
$$


Нетрудно видеть, что

$$
\lim _{\substack{x \rightarrow 0 \\ y \rightarrow y_{0} \\ z \rightarrow z_{0}}} \frac{1+2 \alpha}{2 \pi} x^{1+2 \alpha} \int_{p}^{q} d \eta \int_{h(\eta)}^{k(\eta)}\left[x^{2}+(\eta-y)^{2}+(\zeta-z)^{2}\right]^{-3 / 2-\alpha} d \zeta=1 .
$$

Действительно, полагая $\eta=y+x s$ и $\zeta=z+x t$, будем иметь

$$
\begin{aligned}
& \frac{1+2 \alpha}{2 \pi} x^{1+2 \alpha} \int_{p}^{q} d \eta \int_{h(\eta)}^{k(\eta)} \nu_{1}(\eta, \zeta)\left[x^{2}+(\eta-y)^{2}+(\zeta-z)^{2}\right]^{-3 / 2-\alpha} d \zeta= \\
& =\frac{1+2 \alpha}{2 \pi} \int_{(p-y) / x}^{(q-y) / x} \int_{(h(y+x)-z) / x}^{(k(y+x)-z) / x} \nu_{1}(y+x s, z+x t)\left[1+s^{2}+t^{2}\right]^{-3 / 2-\alpha} d s d t .
\end{aligned}
$$

Отсюда, учитывая формулу (35) при $x \rightarrow 0, y \rightarrow y_{0}, z \rightarrow z_{0},\left(y_{0}, z_{0}\right) \in X$, получим (57).

Далее, следуя [17, гл. $2, \S 6]$ и используя равенство (57), можно доказать, что производная $\partial v / \partial x$ с весом $x^{2 \alpha}$ в области $X$ принимает значение $\nu_{1}(y, z)$, т.е.

$$
\lim _{\substack{x \rightarrow 0 \\ y \rightarrow y_{0} \\ z \rightarrow z_{0}}}\left(x^{2 \alpha} \frac{\partial v(x, y, z)}{\partial x}\right)=\nu_{1}\left(y_{0}, z_{0}\right), \quad\left(y_{0}, z_{0}\right) \in X .
$$

Нетрудно видеть, что значения функции $v(x, y, z)$ непрерывны и ограничены на поверхности Г. Теперь решение первоначальной задачи Хольмгрена может быть представлено в виде

$$
u(x, y, z)=v(x, y, z)+w(x, y, z)
$$

где $w(x, y, z)$ - решение уравнения (3) в области $D$, удовлетворяющее краевым условиям

$$
\left.w\right|_{\Gamma}=\varphi(s, t)-\left.v\right|_{\Gamma}=\varphi_{1}(s, t) ;\left.\quad\left(x^{2 \alpha} \frac{\partial w}{\partial x}\right)\right|_{x=0}=0, \quad(y, z) \in X .
$$

Итак, доказано, что в случае задачи Хольмгрена можно ограничиться случаем $\nu_{1}(y, z) \equiv 0$.

5.5. Сведение задачи Хольмгрена к интегральному уравнению. Решение задачи Хольмгрена будем искать в виде потенциала двойного слоя с неизвестной плотностью $\mu(\theta, \vartheta)$ :

$$
w(x, y, z)=\iint_{\Gamma} \mu(\theta, \vartheta) B_{\nu}^{\alpha}[q(\xi, \eta, \zeta ; x, y, z)] d \theta d \vartheta
$$

Потенциал двойного слоя $w(x, y, z)$ удовлетворяет уравнению (3) внутри области $D$ и $\left.\left(x^{2 \alpha} \frac{\partial w}{\partial x}\right)\right|_{x=0}=0$ при $(y, z) \in X$. Для выполнения краевого условия (58) на $Г$ необходимо, чтобы предельные значения $w(x, y, z)$ изнутри равнялись $\varphi_{1}(s, t)$ :

$$
w_{i}(x, y, z)=\varphi_{1}(s, t), \quad(x, y, z) \in \Gamma
$$


Пользуясь первой из формул $(40)$, получим для плотности $\mu(\theta, \vartheta)$ интегральное уравнение

$$
\mu(s, t)-2 \iint_{\Gamma} K(s, t ; \theta, \vartheta) \mu(\theta, \vartheta) d \theta d \vartheta=-2 \varphi_{1}(s, t),
$$

где

$$
K(s, t ; \theta, \vartheta)=B_{\nu}^{\alpha}[q(\xi(\theta, \vartheta), \eta(\theta, \vartheta), \zeta(\theta, \vartheta) ; x(s, t), y(s, t), z(s, t))] .
$$

Как это следует из оценки (39), ядро $K(s, t ; \theta, \vartheta)$ имеет слабую особенность.

Отметим, что если уравнение (59) разрешимо, то его решение непрерывно. Это следует из непрерывности свободного члена и из вида ядра $K(s, t ; \theta, \vartheta)$.

В п. 4 было показано, что $\lambda=2$ не является собственным значением ядра $K(s, t ; \theta, \vartheta)$ и, следовательно, уравнение (59) при любом свободном члене имеют единственное решение. Таким образом, если поверхность Г удовлетворяет условиям 1) и 2) п. 2 и заданные значения решения на поверхности $\Gamma$ непрерывны, то задача Хольмгрена для уравнения (3) имеет единственное решение, и это решение можно представить в виде потенциала двойного слоя.

\section{6. Функция Грина оператора $E(u)$}

\section{1. Функция Грина задачи Хольмгрена.}

ОПРеДЕЛЕНиЕ 3. Функиией Грина задачи Холъмгрена для уравнения (3) называется функция $G\left(x, y, z ; x_{0}, y_{0}, z_{0}\right)$, удовлетворяющая следующим условиям:

1) внутри области $D$, кроме точки $\left(x_{0}, y_{0}, z_{0}\right)$, эта функция есть регулярное решение уравнения (3);

2) она удовлетворяет граничным условиям

$$
\left.G\left(x, y, z ; x_{0}, y_{0}, z_{0}\right)\right|_{\Gamma}=0 ;\left.\quad\left(x^{2 \alpha} \frac{\partial G}{\partial x}\right)\right|_{x=0}=0
$$

3) она может быть представлена в виде

$$
G\left(x, y, z ; x_{0}, y_{0}, z_{0}\right)=q\left(x, y, z ; x_{0}, y_{0}, z_{0}\right)+v\left(x, y, z ; x_{0}, y_{0}, z_{0}\right),
$$

где $q(x, y, z ; \xi, \eta, \zeta)$ - фундаментальное решение уравнения (3), определенное формулой $(6)$, а $v\left(x, y, z ; x_{0}, y_{0}, z_{0}\right)$ - регулярное решение уравнения (3) везде внутри $D$.

Построение функции Грина сводится к нахождению ее регулярной части $v\left(x, y, z ; x_{0}, y_{0}, z_{0}\right)$, которая в силу $(7),(60)$ и (61) должна удовлетворять граничным условиям

$$
\begin{gathered}
\left.v\left(x, y, z ; x_{0}, y_{0}, z_{0}\right)\right|_{\Gamma}=-\left.q\left(x, y, z ; x_{0}, y_{0}, z_{0}\right)\right|_{\Gamma} \\
\left.\left(x^{2 \alpha} \frac{\partial v\left(x, y, z ; x_{0}, y_{0}, z_{0}\right)}{\partial x}\right)\right|_{x=0}=0 .
\end{gathered}
$$

Функцию $v\left(x, y, z ; x_{0}, y_{0}, z_{0}\right)$ будем искать в виде потенциала двойного слоя:

$$
v\left(x, y, z ; x_{0}, y_{0}, z_{0}\right)=\iint_{\Gamma} \mu_{1}\left(\theta, \vartheta ; x_{0}, y_{0}, z_{0}\right) B_{\nu}^{\alpha}[q(\xi, \eta, \zeta ; x, y, z)] d \theta d \vartheta
$$


Принимая во внимание первое из равенств (40) и граничное условие (62), получим интегральное уравнение для плотности $\mu\left(s, t ; x_{0}, y_{0}, z_{0}\right)$ :

$$
\begin{aligned}
\mu\left(s, t ; x_{0}, y_{0}, z_{0}\right)-2 \iint_{\Gamma} K(s, t ; \theta, \vartheta) \mu & \left(\theta, \vartheta ; x_{0}, y_{0}, z_{0}\right) d \theta d \vartheta= \\
& =2 q\left[x(s, t), y(s, t), z(s, t) ; x_{0}, y_{0}, z_{0}\right] .
\end{aligned}
$$

Правая часть уравнения (64) есть непрерывная функция от $s$ и $t$ (точка $\left(x_{0}, y_{0}, z_{0}\right)$ лежит внутри $\left.D\right)$. В п. 4 было доказано, что $\lambda=2$ не является собственным значением ядра $K(s, t ; \theta, \vartheta)$ и, следовательно, уравнение (64) разрешимо и его непрерывное решение можно записать в виде

$$
\begin{aligned}
\mu\left(s, t ; x_{0}, y_{0}, z_{0}\right)=2 q[x(s, t), & \left.y(s, t), z(s, t) ; x_{0}, y_{0}, z_{0}\right]+ \\
& +4 \iint_{\Gamma} R(s, t ; \theta, \vartheta ; 2) q\left(\xi, \eta, \zeta ; x_{0}, y_{0}, z_{0}\right) d \theta d \vartheta
\end{aligned}
$$

где $R(s, t ; \theta, \vartheta ; 2)$ - резольвента ядра $K(s, t ; \theta, \vartheta) ;(x(s, t), y(s, t), z(s, t)) \in \Gamma$. Подставляя (65) в (63), получим

$$
\begin{aligned}
& v\left(x, y, z ; x_{0}, y_{0}, z_{0}\right)=2 \iint_{\Gamma} q\left(\xi, \eta, \zeta ; x_{0}, y_{0}, z_{0}\right) B_{\nu}^{\alpha}[q(\xi, \eta, \zeta ; x, y, z)] d \theta d \vartheta+ \\
&+4 \iint_{\Gamma} \iint_{\Gamma} B_{\nu}^{\alpha}[q(\xi, \eta, \zeta ; x, y, z)] R(\theta, \vartheta ; s, t ; 2) \times \\
& \quad \times q\left[x(s, t), y(s, t), z(s, t) ; x_{0}, y_{0}, z_{0}\right] d \theta d \vartheta d s d t .
\end{aligned}
$$

Определим теперь функцию

$$
g(x, y, z)=\left\{\begin{array}{rr}
v\left(x, y, z ; x_{0}, y_{0}, z_{0}\right), & (x, y, z) \in D \\
-q\left(x, y, z ; x_{0}, y_{0}, z_{0}\right), & (x, y, z) \in D^{\prime}
\end{array}\right.
$$

Функция $g(x, y, z)$ является регулярным решением уравнения (3) как внутри области $D$, так и внутри $D^{\prime}$ и равна нулю на бесконечности. Так как точка $\left(x_{0}, y_{0}, z_{0}\right)$ лежит внутри $D$, то в $D^{\prime}$ функция $g(x, y, z)$ имеет производные любого порядка, непрерывные вплоть до Г. Мы можем рассматривать $g(x, y, z)$ в $D^{\prime}$ как решение уравнения (3), удовлетворяющее граничным условиям

$$
\begin{gathered}
\left.B_{n}^{\alpha}[g(x, y, z)]\right|_{\Gamma}=-B_{n}^{\alpha}\left[q\left(x(s, t), y(s, t), z(s, t) ; x_{0}, y_{0}, z_{0}\right)\right], \\
\left.\left(x^{2 \alpha} \frac{\partial g(x, y, z)}{\partial x}\right)\right|_{x=0}=0 .
\end{gathered}
$$

Это решение представим в виде потенциала простого слоя

$$
g(x, y, z)=\iint_{\Gamma} \rho\left(\theta, \vartheta ; x_{0}, y_{0}, z_{0}\right) q(\xi, \eta, \zeta ; x, y, z) d \theta d \vartheta, \quad(x, y, z) \in \mathbb{R}_{3}^{+} \backslash \bar{D}
$$

с неизвестной плотностью $\rho\left(\theta, \vartheta ; x_{0}, y_{0}, z_{0}\right)$. 
Воспользовавшись второй из формул (44), получим интегральное уравнение для плотности $\rho\left(s, t ; x_{0}, y_{0}, z_{0}\right)$ :

$$
\begin{aligned}
& \rho\left(s, t ; x_{0}, y_{0}, z_{0}\right)-2 \iint_{\Gamma} K(\theta, \vartheta ; s, t) \rho\left(\theta, \vartheta ; x_{0}, y_{0}, z_{0}\right) d \theta d \vartheta= \\
& \quad=2 B_{n}^{\alpha}\left[q\left(x(s, t), y(s, t), z(s, t) ; x_{0}, y_{0}, z_{0}\right)\right] .
\end{aligned}
$$

Уравнение (69) - союзное с уравнением (64). Его правая часть есть непрерывная функция от $s$ и $t$. Таким образом, уравнение (69) имеет непрерывное решение:

$$
\begin{aligned}
\rho\left(s, t ; x_{0}, y_{0}, z_{0}\right)=2 B_{n}^{\alpha}[ & \left.q\left(x(s, t), y(s, t), z(s, t) ; x_{0}, y_{0}, z_{0}\right)\right] \\
& +4 \iint_{\Gamma} R(\theta, \vartheta ; s, t ; 2) B_{\nu}^{\alpha}\left[q\left(\xi, \eta, \zeta ; x_{0}, y_{0}, z_{0}\right)\right] d \theta d \vartheta .
\end{aligned}
$$

Значения потенциала простого слоя $g(x, y, z)$ на поверхности $\Gamma$ равны $-q\left(x, y, z ; x_{0}, y_{0}, z_{0}\right)$, т.е. такие же, как и функции $v\left(x, y, z ; x_{0}, y_{0}, z_{0}\right)$, а на плоскости $y O z$ их частные производные по $x$ равны нулю. Отсюда в силу теоремы единственности задачи Хольмгрена следует, что формула (68) для функции $g(x, y, z)$, определенной равенством (67), справедлива во всем полупространстве $x \geqslant 0$, т.е.

$$
\begin{aligned}
& v\left(x, y, z ; x_{0}, y_{0}, z_{0}\right)= \\
& \quad=\iint_{\Gamma} \rho\left(\theta, \vartheta ; x_{0}, y_{0}, z_{0}\right) q(\xi, \eta, \zeta ; x, y, z) d \theta d \vartheta, \quad(x, y, z) \in D .
\end{aligned}
$$

Таким образом, регулярная часть $v\left(x, y, z ; x_{0}, y_{0}, z_{0}\right)$ функции Грина представима в виде потенциала простого слоя.

Применяя первую из формул (44) к (71), получим

$$
\begin{aligned}
2 B_{n}^{\alpha}\left[v\left(x(s, t), y(s, t), z(s, t) ; x_{0}, y_{0}, z_{0}\right)\right]_{i} & = \\
& =\rho\left(s, t ; x_{0}, y_{0}, z_{0}\right)+2 \iint_{\Gamma} K(\theta, \vartheta ; s, t) \rho\left(\theta, \vartheta ; x_{0}, y_{0}, z_{0}\right) d \theta d \vartheta
\end{aligned}
$$

но, согласно (69), имеем

$$
\begin{aligned}
& 2 B_{n}^{\alpha}\left[q\left(x(s, t), y(s, t), z(s, t) ; x_{0}, y_{0}, z_{0}\right)\right]_{i}= \\
& \quad=\rho\left(s, t ; x_{0}, y_{0}, z_{0}\right)-2 \iint_{\Gamma} K(\theta, \vartheta ; s, t) \rho\left(\theta, \vartheta ; x_{0}, y_{0}, z_{0}\right) d \theta d \vartheta .
\end{aligned}
$$

Складывая почленно последние два равенства и принимая во внимание (61), будем иметь

$$
B_{n}^{\alpha}\left[G\left(x(s, t), y(s, t), z(s, t) ; x_{0}, y_{0}, z_{0}\right)\right]=\rho\left(s, t ; x_{0}, y_{0}, z_{0}\right),
$$

и, следовательно, формулу (71) можно записать в виде

$$
v\left(x, y, z ; x_{0}, y_{0}, z_{0}\right)=\iint_{\Gamma} B_{\nu}^{\alpha}\left[G\left(\xi, \eta, \zeta ; x_{0}, y_{0}, z_{0}\right)\right] q(\xi, \eta, \zeta ; x, y, z) d \theta d \vartheta
$$


Умножая теперь обе части равенства (70) на $q(x(s, t), y(s, t), z(s, t) ; x, y, z)$, интегрируя по Г и учитывая (65) и (63), получим

$$
v\left(x_{0}, y_{0}, z_{0} ; x, y, z\right)=\iint_{\Gamma} \rho\left(\theta, \vartheta ; x_{0}, y_{0}, z_{0}\right) q(\xi, \eta, \zeta ; x, y, z) d \theta d \vartheta
$$

Сравнивая это с формулой (71), будем иметь

$$
v\left(x, y, z ; x_{0}, y_{0}, z_{0}\right)=v\left(x_{0}, y_{0}, z_{0} ; x, y, z\right)
$$

если точки $(x, y, z)$ и $\left(x_{0}, y_{0}, z_{0}\right)$ находятся внутри области $D$.

Лемма 4. Функиия Грина $G\left(x, y, z ; x_{0}, y_{0}, z_{0}\right)$ симметрична относительно точек $(x, y, z)$ u $\left(x_{0}, y_{0}, z_{0}\right)$, если они находятся внутри области $D$.

Доказательст во леммы 4 следует из представления (61) функции Грина и равенства (73).

Для области $D_{0}$, ограниченной кругом $y^{2}+z^{2} \leqslant a^{2}$ плоскости $y O z$ и полусферой $x^{2}+y^{2}+z^{2}=a^{2}, x \geqslant 0$, функция Грина задачи Хольмгрена имеет вид

$$
G_{0}\left(x, y, z ; x_{0}, y_{0}, z_{0}\right)=q\left(x, y, z ; x_{0}, y_{0}, z_{0}\right)-\left(\frac{a}{R}\right)^{1+2 \alpha} q\left(x, y, z ; \tilde{x}_{0}, \tilde{y}_{0}, \tilde{z}_{0}\right),
$$

где

$$
R^{2}=x_{0}^{2}+y_{0}^{2}+z_{0}^{2}, \quad \tilde{x}_{0}=\frac{a^{2}}{R^{2}} x_{0}, \quad \tilde{y}_{0}=\frac{a^{2}}{R^{2}} y_{0}, \quad \tilde{z}_{0}=\frac{a^{2}}{R^{2}} z_{0} .
$$

Покажем, что регулярную часть

$$
v_{0}\left(x, y, z ; x_{0}, y_{0}, z_{0}\right)=-\left(\frac{a}{R}\right)^{1+2 \alpha} q\left(x, y, z ; \tilde{x}_{0}, \tilde{y}_{0}, \tilde{z}_{0}\right)
$$

функции Грина $G_{0}\left(x, y, z ; x_{0}, y_{0}, z_{0}\right)$ можно представить в виде

$$
\begin{aligned}
& v_{0}\left(x, y, z ; x_{0}, y_{0}, z_{0}\right)= \\
& =-\iint_{\Gamma} \rho(s, t ; x, y, z) v_{0}\left(x(s, t), y(s, t), z(s, t) ; x_{0}, y_{0}, z_{0}\right) d s d t
\end{aligned}
$$

где $\rho(s, t ; x, y, z)$ - решение уравнения $(71)$.

Действительно, пусть $\left(x_{0}, y_{0}, z_{0}\right)$ - произвольная точка внутри области $D$. Рассмотрим функцию

$$
u\left(x, y, z ; x_{0}, y_{0}, z_{0}\right)=-\iint_{\Gamma} \rho(s, t ; x, y, z) v_{0}\left(x(s, t), y(s, t), z(s, t) ; x_{0}, y_{0}, z_{0}\right) d s d t
$$

Она, как функция от $(x, y, z)$, удовлетворяет уравнению $(3)$, так как этому уравнению удовлетворяет функция $\rho(s, t ; x, y, z)$. Подставляя вместо $\rho(s, t ; x, y, z)$ ее выражение $(70)$, получим 


$$
\begin{aligned}
& u\left(x, y, z ; x_{0}, y_{0}, z_{0}\right)= \\
& \quad=-\iint_{\Gamma} \psi\left(s, t ; x_{0}, y_{0}, z_{0}\right) B_{n}^{\alpha}[q(x(s, t), y(s, t), z(s, t) ; x, y, z)] d s d t
\end{aligned}
$$

где

$$
\begin{aligned}
\psi\left(s, t ; x_{0}, y_{0}, z_{0}\right)=2 v_{0}(x(s, t), y & \left.(s, t), z(s, t) ; x_{0}, y_{0}, z_{0}\right)+ \\
& +4 \iint_{\Gamma} R(s, t ; \theta, \vartheta ; 2) v_{0}\left(\xi, \eta, \zeta ; x_{0}, y_{0}, z_{0}\right) d \theta d \vartheta
\end{aligned}
$$

т.е. $\psi\left(s, t ; x_{0}, y_{0}, z_{0}\right)$ - решение интегрального уравнения

$$
\begin{aligned}
\psi\left(s, t ; x_{0}, y_{0}, z_{0}\right)-2 \iint_{\Gamma} K(s, t ; \theta, \vartheta) & \psi\left(\theta, \vartheta ; x_{0}, y_{0}, z_{0}\right) d \theta d \vartheta= \\
& =2 v_{0}\left(x(s, t), y(s, t), z(s, t) ; x_{0}, y_{0}, z_{0}\right) .
\end{aligned}
$$

Применяя первую из формул (40) к потенциалу двойного слоя (76), получим

$$
\begin{aligned}
u_{i}(x(s, t), y(s, t), & \left.z(s, t) ; x_{0}, y_{0}, z_{0}\right)= \\
= & \frac{1}{2} \psi\left(s, t ; x_{0}, y_{0}, z_{0}\right)-\iint_{\Gamma} K(s, t ; \theta, \vartheta) \psi\left(\theta, \vartheta ; x_{0}, y_{0}, z_{0}\right) d \theta d \vartheta
\end{aligned}
$$

откуда в силу (77) имеем

$$
\begin{aligned}
u_{i}(x(s, t), & \left.y(s, t), z(s, t) ; x_{0}, y_{0}, z_{0}\right)= \\
& =v_{0}\left(x(s, t), y(s, t), z(s, t) ; x_{0}, y_{0}, z_{0}\right), \quad(x(s, t), y(s, t), z(s, t)) \in \Gamma .
\end{aligned}
$$

Нетрудно убедиться, что

$$
\left.\left(x^{2 \alpha} \frac{\partial u\left(x, y, z ; x_{0}, y_{0}, z_{0}\right)}{\partial x}\right)\right|_{x=0}=0,\left.\quad\left(x^{2 \alpha} \frac{\partial v_{0}\left(x, y, z ; x_{0}, y_{0}, z_{0}\right)}{\partial x}\right)\right|_{x=0}=0 .
$$

Таким образом, функции $u\left(x, y, z ; x_{0}, y_{0}, z_{0}\right)$ и $v_{0}\left(x, y, z ; x_{0}, y_{0}, z_{0}\right)$ удовлетворяют одному и тому же уравнению (3) и одинаковым краевым условиям, и в силу единственности решения задачи Хольмгрена

$$
u\left(x, y, z ; x_{0}, y_{0}, z_{0}\right) \equiv v_{0}\left(x, y, z ; x_{0}, y_{0}, z_{0}\right)
$$

Вычитая теперь из (61) обе части равенства (74), получим

$$
\begin{array}{r}
H\left(x, y, z ; x_{0}, y_{0}, z_{0}\right)=G\left(x, y, z ; x_{0}, y_{0}, z_{0}\right)-G_{0}\left(x, y, z ; x_{0}, y_{0}, z_{0}\right)= \\
=v\left(x, y, z ; x_{0}, y_{0}, z_{0}\right)-v_{0}\left(x, y, z ; x_{0}, y_{0}, z_{0}\right)
\end{array}
$$

или в силу $(71),(73),(74)$ и (75)

$$
H\left(x, y, z ; x_{0}, y_{0}, z_{0}\right)=\iint_{\Gamma} \rho(\theta, \vartheta ; x, y, z) G_{0}\left(\xi, \eta, \zeta ; x_{0}, y_{0}, z_{0}\right) d \theta d \vartheta
$$




\section{2. Решение задачи Хольмгрена для уравнения (3).}

Пусть $\left(x_{0}, y_{0}, z_{0}\right)$ - точка внутри области $D$. Рассмотрим область $D_{\varepsilon, \delta} \subset D$, ограниченную поверхностью $\Gamma_{\varepsilon}$, параллельной поверхности $\Gamma$, и областью $X_{\varepsilon, \delta}$, лежащей на плоскости $x=\delta>\varepsilon$. Выберем $\varepsilon$ и $\delta$ столь малыми, чтобы точка $\left(x_{0}, y_{0}, z_{0}\right)$ находилась внутри $D_{\varepsilon, \delta}$. Вырежем из области $D_{\varepsilon, \delta}$ шар малого радиуса $\rho$ с центром в точке $\left(x_{0}, y_{0}, z_{0}\right)$ и оставшуюся часть $D_{\varepsilon, \delta}$ обозначим через $D_{\varepsilon, \delta}^{\rho}$, в которой функция Грина $G\left(x, y, z ; x_{0}, y_{0}, z_{0}\right)$ будет регулярным решением уравнения (3).

Пусть $u(x, y, z)$ есть регулярное решение уравнения (3) в области $D$, удовлетворяющее граничным условиям (54).

Применяя формулу (11), получим

$$
\begin{aligned}
\iint_{\Gamma_{\varepsilon}}\left(G B_{n}^{\alpha}[u]-u B_{n}^{\alpha}[G]\right) d s d t+\left.\iint_{X_{\varepsilon, \delta}} x^{2 \alpha}\left(u \frac{\partial G}{\partial x}-G \frac{\partial u}{\partial x}\right)\right|_{x=\delta} d y d z= \\
\quad=\iint_{C_{\rho}}\left(G B_{n}^{\alpha}[u]-u B_{n}^{\alpha}[G]\right) d s d t
\end{aligned}
$$

где $C_{\rho}$ - сфера вырезанного шара. Переходя к пределу при $\rho \rightarrow 0$, а затем при $\varepsilon \rightarrow 0$ и $\delta \rightarrow 0$, получим

$$
\begin{aligned}
u\left(x_{0}, y_{0}, z_{0}\right)=-\iint_{X} \nu_{1}(y, z) G\left(0, y, z ; x_{0}, y_{0}, z_{0}\right) d y d z- & \\
& -\iint_{\Gamma} \varphi(\theta, \vartheta) B_{n}^{\alpha}\left[G\left(\xi, \eta, \zeta ; x_{0}, y_{0}, z_{0}\right)\right] d \theta d \vartheta .
\end{aligned}
$$

Покажем, что формула (79) дает решение задачи Хольмгрена.

Нетрудно видеть, что первый интеграл $I_{1}\left(x_{0}, y_{0}, z_{0}\right)$ в формуле $(79)$ есть регулярное в области $D$ решение уравнения $(3)$, непрерывное в $\bar{D}$. Обозначим

$$
\begin{aligned}
\varphi\left(x_{0}, y_{0}, z_{0}\right)=\iint_{X} \nu_{1}(y, z) q\left(0, y, z ; x_{0}, y_{0}, z_{0}\right) d y d z= \\
\quad=\frac{1}{2 \pi} \iint_{X} \nu_{1}(y, z)\left[x_{0}^{2}+\left(y-y_{0}\right)^{2}+\left(z-z_{0}\right)^{2}\right]^{-1 / 2-\alpha} d y d z .
\end{aligned}
$$

Легко видеть, что $\varphi\left(x_{0}, y_{0}, z_{0}\right)$ есть непрерывная функция в $\bar{D}$. Интеграл $I_{1}\left(x_{0}, y_{0}, z_{0}\right)$ в силу $(80)$ и (66) и симметричности функции $v\left(x, y, z ; x_{0}, y_{0}, z_{0}\right)$ можно представить в виде

$$
\begin{aligned}
& I_{1}\left(x_{0}, y_{0}, z_{0}\right)=-\varphi\left(x_{0}, y_{0}, z_{0}\right)-2 \iint_{\Gamma} \phi(\xi, \eta, \zeta) B_{\nu}^{\alpha}\left[q\left(\xi, \eta, \zeta ; x_{0}, y_{0}, z_{0}\right)\right] d \theta d \vartheta- \\
&-4 \iint_{\Gamma} \iint_{\Gamma} R(\theta, \vartheta ; s, t ; 2) \varphi(x(s, t), y(s, t), z(s, t)) \times \\
& \quad \times B_{\nu}^{\alpha}\left[q\left(\xi, \eta, \zeta ; x_{0}, y_{0}, z_{0}\right)\right] d \theta d \vartheta d s d t
\end{aligned}
$$


Последние два интеграла в формуле (81) - потенциалы двойного слоя. Принимая во внимание первую из формул (40) и интегральное уравнение для резольвенты $R(s, t ; \theta, \vartheta ; 2)$, из формулы (81) получим

$$
\left.I_{1}\left(x_{0}, y_{0}, z_{0}\right)\right|_{\Gamma}=0 .
$$

Нетрудно видеть, что

$$
\lim _{x_{0} \rightarrow 0} x_{0}^{2 \alpha} \frac{\partial I_{1}\left(x_{0}, y_{0}, z_{0}\right)}{\partial x_{0}}=\nu_{1}\left(y_{0}, z_{0}\right), \quad\left(y_{0}, z_{0}\right) \in X .
$$

В самом деле, интеграл $I_{1}\left(x_{0}, y_{0}, z_{0}\right)$ в силу $(71)$ и симметричности функции $v\left(x, y, z ; x_{0}, y_{0}, z_{0}\right)$ можно записать также в виде

$$
\begin{aligned}
& I_{1}\left(x_{0}, y_{0}, z_{0}\right)=-\iint_{X} \nu_{1}(y, z) q\left(0, y, z ; x_{0}, y_{0}, z_{0}\right) d y d z- \\
& \quad-\iint_{X} \nu_{1}(y, z) d y d z \iint_{\Gamma} \rho(\theta, \vartheta ; 0, y, z) q\left(\xi, \eta, \zeta ; x_{0}, y_{0}, z_{0}\right) d \theta d \vartheta .
\end{aligned}
$$

В п. 5 показано, что производная по $x_{0}$ от первого слагаемого, умноженного на $x_{0}^{2 \alpha}$, равна $\nu_{1}\left(y_{0}, z_{0}\right)$ при $x_{0} \rightarrow 0,\left(y_{0}, z_{0}\right) \in X$. Производная по $x_{0}$ от второго слагаемого, умноженного на $x_{0}^{2 \alpha}$, равна нулю при $x_{0}=0$, так как $x_{0}^{2 \alpha} \frac{\partial q}{\partial x_{0}}=0$ при $x_{0} \rightarrow 0,\left(y_{0}, z_{0}\right) \in X$.

Рассмотрим второй интеграл $I_{2}\left(x_{0}, y_{0}, z_{0}\right)$ в формуле $(79)$, который в силу (70) и (72) можно записать в виде

$$
\begin{aligned}
& I_{2}\left(x_{0}, y_{0}, z_{0}\right)=-\iint_{\Gamma} \varphi(s, t) \rho\left(s, t ; x_{0}, y_{0}, z_{0}\right) d s d t= \\
& \quad=-\iint_{\Gamma} \chi(\theta, \vartheta) B_{\nu}^{\alpha}\left[q\left(\xi, \eta, \zeta ; x_{0}, y_{0}, z_{0}\right)\right] d \theta d \vartheta
\end{aligned}
$$

где

$$
\chi(\theta, \vartheta)=2 \varphi(\theta, \vartheta)+4 \iint_{\Gamma} R(\theta, \vartheta ; s, t ; 2) \varphi(s, t) d s d t
$$

т.е. функция $\chi(\theta, \vartheta)$ - решение интегрального уравнения

$$
\chi(s, t)-2 \iint_{\Gamma} K(s, t) \chi(\theta, \vartheta) d \theta d \vartheta=2 \varphi(s, t) .
$$

Так как $\chi(s, t)$ - непрерывная функция, $I_{2}\left(x_{0}, y_{0}, z_{0}\right)$ - регулярное в области $D$ решение уравнения (3), непрерывное в $\bar{D}$, которое в силу $(40)$ и $(82)$ удовлетворяет условию

$$
\left.I_{2}\left(x_{0}, y_{0}, z_{0}\right)\right|_{\Gamma}=\varphi(s, t)
$$

нетрудно видеть, что

$$
\lim _{x_{0} \rightarrow 0} x_{0}^{2 \alpha} \frac{\partial I_{2}\left(x_{0}, y_{0}, z_{0}\right)}{\partial x_{0}}=0, \quad\left(y_{0}, z_{0}\right) \in X
$$


Используя формулы (78) и (74), решение (79) задачи Хольмгрена для уравнения (3) можно записать в виде

$$
\begin{aligned}
& u\left(x_{0}, y_{0}, z_{0}\right)= \\
& =-\iint_{\Gamma} \varphi(\theta, \vartheta)\left\{B_{\nu}^{\alpha}\left[G_{0}\left(\xi, \eta, \zeta ; x_{0}, y_{0}, z_{0}\right)\right]+B_{\nu}^{\alpha}\left[H\left(\xi, \eta, \zeta ; x_{0}, y_{0}, z_{0}\right)\right]\right\} d \theta d \vartheta- \\
& \quad-\iint_{X} \nu_{1}(y, z)\left[G_{0}\left(0, y, z ; x_{0}, y_{0}, z_{0}\right)+H\left(0, y, z ; x_{0}, y_{0}, z_{0}\right)\right] d y d z,
\end{aligned}
$$

где $G_{0}\left(\xi, \eta, \zeta ; x_{0}, y_{0}, z_{0}\right)$ - функция Грина задачи Хольмгрена для полусферической области $D_{0}$ :

$$
\begin{aligned}
& G_{0}\left(\xi, \eta, \zeta ; x_{0}, y_{0}, z_{0}\right)=\frac{1}{2 \pi} \frac{1}{r^{1+2 \alpha}} F\left(\alpha+\frac{1}{2}, \alpha ; 2 \alpha ; 1-\frac{r_{1}^{2}}{r^{2}}\right)- \\
& -\frac{1}{2 \pi} \frac{1}{\tilde{r}^{1+2 \alpha}}\left(\frac{a}{R}\right)^{1+2 \alpha} F\left(\alpha+\frac{1}{2}, \alpha ; 2 \alpha ; 1-\frac{\tilde{r}_{1}^{2}}{\tilde{r}^{2}}\right), \\
& a^{2}=\xi^{2}+\eta^{2}+\zeta^{2}, \quad R^{2}=x_{0}^{2}+y_{0}^{2}+z_{0}^{2}, \\
& \left.\begin{array}{l}
r_{1}^{2} \\
r^{2}
\end{array}\right\}=\left(\xi \pm x_{0}\right)^{2}+\left(\eta-y_{0}\right)^{2}+\left(\zeta-z_{0}\right)^{2}, \\
& \left.\begin{array}{c}
\tilde{r}_{1}^{2} \\
\tilde{r}^{2}
\end{array}\right\}=\left(\xi \pm \frac{a^{2}}{R^{2}} x_{0}\right)^{2}+\left(\eta-\frac{a^{2}}{R^{2}} y_{0}\right)^{2}+\left(\zeta-\frac{a^{2}}{R^{2}} z_{0}\right)^{2}
\end{aligned}
$$

и

$$
H\left(x, y, z ; x_{0}, y_{0}, z_{0}\right)=\iint_{\Gamma} \rho\left(\theta, \vartheta ; x_{0}, y_{0}, z_{0}\right) G_{0}(\xi, \eta, \zeta ; x, y, z) d \theta d \vartheta .
$$

Непосредственным вычислением можно показать, что функция, определенная формулой (83), является решением задачи Хольмгрена для уравнения (3), т.е. она удовлетворяет уравнению (3) и условиям (54).

6.3. Решение задачи Хольмгрена для полушара. В этом случае функция $H\left(\xi, \eta, \zeta ; x_{0}, y_{0}, z_{0}\right)$ тождественно равна нулю:

$$
H\left(\xi, \eta, \zeta ; x_{0}, y_{0}, z_{0}\right) \equiv 0, \quad(\xi, \eta, \zeta) \in D_{0} .
$$

Чтобы явно получить решение задачи Хольмгрена по формуле (83) для полуферической области $D_{0}$, необходимо вычислить конормальную производную функции Грина (84). Воспользовавшись выведенной выше формулой (21), нетрудно найти аналогичную формулу для $B_{\nu}^{\alpha}\left[q\left(\xi, \eta, \zeta ; \tilde{x}_{0}, \tilde{y}_{0}, \tilde{z}_{0}\right)\right]$. Далее, следуя [26, § 26], имеем

$$
B_{\nu}^{\alpha}\left[G_{0}\left(\xi, \eta, \zeta ; x_{0}, y_{0}, z_{0}\right)\right]=\frac{1+2 \alpha}{2 \pi} \xi^{2 \alpha} F\left(\frac{3}{2}+\alpha, \alpha ; 2 \alpha ; 1-\frac{r_{1}^{2}}{r^{2}}\right) \frac{R^{2}-a^{2}}{a r^{3+2 \alpha}} .
$$

Подставив (85) и (86) в (83), окончательно получим решение задачи Хольмгрена для уравнения (3) в полусферической области $D_{0}$ в явном виде: 


$$
\begin{gathered}
u\left(x_{0}, y_{0}, z_{0}\right)=-\frac{1}{2 \pi} \int_{-a}^{a} d y \int_{-\sqrt{a^{2}-y^{2}}}^{\sqrt{a^{2}-y^{2}}} \nu_{1}(y, z)\left\{\left[x_{0}^{2}+\left(y-y_{0}\right)^{2}+\left(z-z_{0}\right)^{2}\right]^{-1 / 2-\alpha}-\right. \\
\left.-\left[\left(a-\frac{y y_{0}}{a}\right)^{2}+\left(a-\frac{z z_{0}}{a}\right)^{2}+\frac{x_{0}^{2} y^{2}+y^{2} z_{0}^{2}+x_{0}^{2} z^{2}+y_{0}^{2} z^{2}}{a^{2}}-a^{2}\right]^{-1 / 2-\alpha}\right\} d z+ \\
+\frac{1+2 \alpha}{2 \pi} \iint_{\Gamma} \varphi(\theta, \vartheta) \xi^{2 \alpha} F\left(\frac{3}{2}+\alpha, \alpha ; 2 \alpha ; 1-\frac{r_{1}^{2}}{r^{2}}\right) \frac{a^{2}-R^{2}}{a r^{3+2 \alpha}} d \theta d \vartheta .
\end{gathered}
$$

Решение (83) (в частности формула (87)) задачи Хольмгрена более удобно для дальнейших исследований. Действительно, полученные формулы (83) и (87) играют важную роль при изучении краевых задач для уравнений смешанного типа, т.е. для уравнений эллиптико-гиперболического или эллиптико-параболического типов: каждая из этих формул позволяет легко вывести основное функциональное соотношение между следами искомого решения и его производной на линии вырождения, принесенное из эллиптической части смешанной области.

Конкурирующие интересы. У меня нет конфликта интересов в авторстве и публикации этой статьи.

Авторская ответственность. Я несу полную ответственность за предоставление окончательной версии рукописи в печать. Окончательная версия рукописи мною одобрена.

Финансирование. Исследование выполнялось без финансирования.

\section{Библиографический список}

1. Кондратьев Б. П. Теория потенциала. Новъе методы и задачи с решениями. М.: Мир, 2007. 512 c.

2. Ergashev T. G. Fundamental solutions for a class of multidimensional elliptic equations with several singular coefficients // J. Sib. Fed. Univ. Math. Phys., 2020. vol. 13, no. 1. pp. 48-57. https://doi.org/10.17516/1997-1397-2020-13-1-48-57.

3. Ergashev T. G. Fundamental solutions of the generalized Helmholtz equation with several singular coefficients and confluent hypergeometric functions of many variables // Lobachevskii J. Math, 2020. vol.41, no.1. pp. 15-26. https://doi.org/10.1134/ S1995080220010047.

4. Hasanov A. Fundamental solutions bi-axially symmetric Helmholtz equation// Complex Var. Elliptic Equ., 2007. vol.52, no.8. pp. 673-683. https://doi.org/10.1080/ 17476930701300375.

5. Hasanov A., Karimov E. T. Fundamental solutions for a class of three-dimensional elliptic equations with singular coefficients // Appl. Math. Letters, 2009. vol. 22, no.12. pp. 18281832, arXiv: 0901.0468 [math-ph]. https://doi.org/10.1016/j.aml.2009.07.006.

6. Urinov A. K., Karimov E. T. On fundamental solutions for 3D singular elliptic equations with a parameter// Appl. Math. Letters, 2011. vol.24, no.3. pp. 314-319. https://doi. org $/ 10.1016 / \mathrm{j} . \mathrm{aml} \cdot 2010 \cdot 10.013$.

7. Ergashev T. G. On fundamental solutions for multidimensional Helmholtz equation with three singular coefficients // Comp. Math. Appl., 2019. vol.77, no.1. pp. 69-76, arXiv: 1804.04363 [math.AP].

8. Уринов А. К., Эргашев Т. Г. Конфлюэнтные гипергеометрические функции многих переменных и их применение к нахождению фундаментальных решений обобщенного уравнения Гельмгольца с сингулярными коэффициентами // Вестн. Томск. гос. ун-та. Матем. и мех., 2018. №55. C. 45-56. https://doi.org/10.17223/19988621/55/5. 
9. Mavlyaviev R. M., Garipov I. B. Fundamental solution of multidimensional axisymmetric Helmholtz equation // Complex Var. Elliptic Equ., 2017. vol. 63, no. 3. pp. 287-296. https : // doi.org/10.1080/17476933.2016.1218853.

10. Мавлявиев Р. М. Построение фундаментальных решений $B$-эллиптических уравнений с младшими членами // Изв. вузов. Матем., 2017. №6. С. 70-75.

11. Уринов А. К., Каримов К. Т. Задача Дирихле для трехмерного уравнения смешанного типа с тремя сингулярными коэффициентами // Вестн. Сам. гос. техн. ун-та. Сер. Физ.-мат. науки, 2017. Т. 21, № 4. С. 665-683. https://doi.org/10.14498/vsgtu1559.

12. Shishkina E. L. The Dirichlet problem for an elliptic singular equation // Complex Var. Elliptic Equ., 2020. vol. 65, no. 7. pp. 1210-1218. https://doi.org/10.1080/17476933.2019. 1588259.

13. Эргашев Т. Г. Обобщённая задача Хольмгрена для эллиптического уравнения с несколькими сингулярными коэффициентами // Диффер. уравн., 2020. Т. 56, №7. C. 872-886, arXiv: 1910.05264 [math.AP]. https://doi.org/10.1134/S0374064120070043.

14. Gellerstedt S. Sur un problème aux limites pour l'équation $y^{2 s} z_{x x}+z_{y y}=0 / /$ Ark. Mat. Astron. Fys. A, 1935. vol. 25, no. 10. pp. 1-12 (In French).

15. Франкль Ф. И. К теории уравнения $y \frac{\partial^{2} z}{\partial x^{2}}+\frac{\partial^{2} z}{\partial y^{2}}=0 / /$ Изв. АН СССР. Сер. матем., 1946. Т. 10, № 2. С. 135-166.

16. Пулькин С. П. Некоторые краевые задачи для уравнения $u_{x x} \pm u_{y y}+p x^{-1} u_{x}=0 / /$ Уч. зап. Куйбышев. пед. ин-та., 1958. Т. 21. С. 3-55.

17. Смирнов М. М. Вырождающиеся эллиптические и гиперболические уравнения. М.: Наука, 1966. 292 с.

18. Srivastava H. M., Hasanov A., Choi J. Double-layer potentials for a generalized bi-axially symmetric Helmholtz equation// Sohag J. Math., 2015. vol. 2, no.1. pp. 1-10, arXiv: 0810.3979 [math.AP].

19. Berdyshev A. S., Hasanov A., Ergashev T. G. Double-layer potentials for a generalized biaxially symmetric Helmholtz equation. II // Complex Var. Elliptic Equ., 2020. vol. 65, no. 2. pp. 316-332. https://doi.org/10.1080/17476933.2019.1583219.

20. Эргашев Т. Г. Третий потенциал двойного слоя для обобщенного двуосесимметрического уравнения Гельмгольца // Уфимск. матем. журн., 2018. Т. 10, № 4. С. 111-122, arXiv: 1807.00903 [math.AP].

21. Эргашев Т. Г. Четвертый потенциал двойного слоя для обобщенного двуосесимметрического уравнения Гельмгольца // Вестн Том. гос. ун-та. Математика и механика, 2017. № 50. C. 45-56. https://doi.org/10.17223/19988621/50/4.

22. Мухлисов Ф. Г., Нигмедзянова А. М. Решение краевых задач для вырождающегося эллиптического уравнения второго рода методом потенциалов // Изв. вузов. Матем., 2009. № 8. C. 57-70.

23. Ergashev T. G. Potentials for three-dimensional singular equation and their application to the solving a mixed problem// Lobachevskii J. Math., 2020. vol.41, no. 6. pp. 1067-1077. https://doi.org/10.1134/S1995080220060086.

24. Бейтмен Г., Эрдейи А. Высшие трансцендентные функиии. Т. 1: Гипергеометрическая функция. Функции Лежандра. М.: Наука, 1973. 296 с.

25. Градштейн И. С., Рыжик И. М. Таблицы интегралов, сумм, рядов и произведений. М.: Физматлит, 1962. $1100 \mathrm{c.}$

26. Сабитов К. Б. Уравнения математической физики. М.: Физматлит, 2013. 352 с. 
MSC: 35J70, 33C20, 33C65

\title{
Potentials for a three-dimensional elliptic equation with one singular coefficient and their application
}

\author{
T. G. Ergashev $v^{1,2}$
}

1 V. I. Romanovskiy Institute of Mathematics, Uzbekistan Academy of Science,

4-a, Universitetskaya st., Tashkent, 100174, Uzbekistan.

2 Tashkent Institute of Irrigation

and Agricultural Mechanization Engineers,

39, Kari Niyaziy st., Tashkent, 100000, Uzbekistan.

\begin{abstract}
A potential theory for a three-dimensional elliptic equation with one singular coefficient is considered. Double- and simple-layer potentials with unknown density are introduced, which are expressed in terms of the fundamental solution of the mentioned elliptic equation. When studying these potentials, the properties of the Gaussian hypergeometric function are used.

Theorems are proved on the limiting values of the introduced potentials and their conormal derivatives, which make it possible to equivalently reduce boundary value problems for singular elliptic equations to an integral equation of the second kind, to which the Fredholm theory is applicable.

The Holmgren problem is solved for a three-dimensional elliptic equation with one singular coefficient in the domain bounded $x=0$ by the coordinate plane and the Lyapunov surface for $x>0$ as an application of the stated theory. The uniqueness of the solution to the stated problem is proved by the well-known abc method, and existence is proved by the method of the Green's function, the regular part of which is sought in the form of the double-layer potential with an unknown density. The solution to the Holmgren problem is found in a form convenient for further research.
\end{abstract}

Keywords: three-dimensional elliptic equation with one singular coefficient, fundamental solution, potential theory, Green's function, Holmgren problem.

Received: $22^{\text {nd }}$ July, 2020 / Revised: $4^{\text {th }}$ February, $2021 /$

Accepted: $11^{\text {th }}$ May, $2021 /$ First online: $11^{\text {th }}$ June, 2021

Competing interests. I hereby declare that I have no conflict of interest in the authorship and publication of this article.

\section{Research Article}

○ () (i) The content is published under the terms of the Creative Commons Attribution 4.0 International License (http://creativecommons.org/licenses/by/4.0/)

Please cite this article in press as:

Ergashev T.G. Potentials for a three-dimensional elliptic equation with one singular coefficient and their application, Vestn. Samar. Gos. Tekhn. Univ., Ser. Fiz.-Mat. Nauki [J. Samara State Tech. Univ., Ser. Phys. Math. Sci.], 2021, vol. 25, no. 2, pp. 257-285. https://doi.org/10.14498/vsgtu1810 (In Russian).

\section{Author's Details:}

Tuhtasin G. Ergashev (1D https://orcid.org/0000-0003-3542-8309

Dr. Phys. \& Math. Sci.; Lab. of Differential Equations and Their Applications ${ }^{1}$; Associate Professor; Dept. Higher Mathematics ${ }^{2}$; e-mail: ergashev.tukhtasin@gmail.com 
Author's Responsibilities. I take full responsibility for submitting the final manuscript in print. I approved the final version of the manuscript.

Funding. This research received no external funding.

\section{References}

1. Kondrat'ev B. P. Teoriia potentsiala. Novye metody $i$ zadachi s resheniiami [Theory of Potential. New Methods and Problems with Solutions]. Moscow, Mir, 2007, 512 pp. (In Russian)

2. Ergashev T. G. Fundamental solutions for a class of multidimensional elliptic equations with several singular coefficients, J. Sib. Fed. Univ. Math. Phys., 2020, vol. 13, no. 1, pp. 48-57. https://doi.org/10.17516/1997-1397-2020-13-1-48-57.

3. Ergashev T. G. Fundamental solutions of the generalized Helmholtz equation with several singular coefficients and confluent hypergeometric functions of many variables, Lobachevskii J. Math, 2020, vol.41, no.1, pp. 15-26. https://doi.org/10.1134/S1995080220010047.

4. Hasanov A. Fundamental solutions bi-axially symmetric Helmholtz equation, Complex Var. Elliptic Equ., 2007, vol.52, no.8, pp. 673-683. https://doi.org/10.1080/ 17476930701300375.

5. Hasanov A., Karimov E. T. Fundamental solutions for a class of three-dimensional elliptic equations with singular coefficients, Appl. Math. Letters, 2009, vol. 22, no. 12, pp. 1828-1832, arXiv: 0901.0468 [math-ph]. https://doi.org/10.1016/j.aml.2009.07.006.

6. Urinov A. K., Karimov E. T. On fundamental solutions for 3D singular elliptic equations with a parameter, Appl. Math. Letters, 2011, vol.24, no. 3, pp. 314-319. https://doi.org/ $10.1016 / j$.aml.2010.10.013.

7. Ergashev T. G. On fundamental solutions for multidimensional Helmholtz equation with three singular coefficients, Comp. Math. Appl., 2019, vol.77, no.1, pp. 69-76, arXiv: 1804.04363 [math.AP].

8. Urinov A. K., Ergashev T. G. Confluent hypergeometric functions of many variables and their application to the finding of fundamental solutions of the generalized helmholtz equation with singular coefficients, Vestn. Tomsk. Gos. Univ. Mat. Mekh., 2018, no. 55, pp. 45-56 (In Russian). https://doi.org/10.17223/19988621/55/5.

9. Mavlyaviev R. M., Garipov I. B. Fundamental solution of multidimensional axisymmetric Helmholtz equation, Complex Var. Elliptic Equ., 2017, vol.63, no. 3, pp. 287-296. https:// doi.org/10.1080/17476933.2016.1218853.

10. Mavlyaviev R. M. Construction of fundamental solutions to $B$-elliptic equations with minor terms, Russian Math. (Iz. VUZ), 2017, vol.61, no. 6, pp. 60-65. https://doi.org/10.3103/ S1066369X17060081.

11. Urinov A. K., Karimov K. T. The Dirichlet problem for a three-dimensional equation of mixed type with three singular coefficients, Vestn. Samar. Gos. Tekhn. Univ., Ser. Fiz.-Mat. Nauki [J. Samara State Tech. Univ., Ser. Phys. Math. Sci.], 2017, vol. 21, no. 4, pp. 665-683 (In Russian). https://doi.org/10.14498/vsgtu1559.

12. Shishkina E. L. The Dirichlet problem for an elliptic singular equation, Complex Var. Elliptic Equ., 2020, vol.65, no. 7, pp. 1210-1218. https://doi.org/10.1080/17476933.2019. 1588259.

13. Ergashev T. G. Generalized Holmgren problem for an elliptic equation with several singular coefficients, Differ. Equ., 2020, vol.56, no.7, pp. 842-856. https://doi.org/ 10.1134/S0012266120070046.

14. Gellerstedt S. Sur un problème aux limites pour l'équation $y^{2 s} z_{x x}+z_{y y}=0$, Ark. Mat. Astron. Fys. A, 1935, vol. 25, no. 10, pp. 1-12 (In French).

15. Frankl F. On the theory of the equation $y \frac{\partial^{2} z}{\partial x^{2}}+\frac{\partial^{2} z}{\partial y^{2}}=0$, Izv. Akad. Nauk SSSR Ser. Mat., 1946, vol. 10, no. 2, pp. 135-166 (In Russian).

16. Pul'kin S. P. Certain boundary value problems for equations $u_{x x} \pm u_{y y}+p x^{-1} u_{x}=0$, Uch. Zap. Kuibyshev. Ped. Inst., 1958, vol. 21, pp. 3-55 (In Russian). 
17. Smirnov M. M. Vyrozhdaiushchiesia ellipticheskie i giperbolicheskie uravneniia [Degenerate Elliptic and Hyperbolic Equations]. Moscow, Nauka, 1966, 292 pp. (In Russian)

18. Srivastava H. M., Hasanov A., Choi J. Double-layer potentials for a generalized bi-axially symmetric Helmholtz equation, Sohag J. Math., 2015, vol. 2, no. 1, pp. 1-10, arXiv: 0810.3979 [math.AP].

19. Berdyshev A. S., Hasanov A., Ergashev T. G. Double-layer potentials for a generalized biaxially symmetric Helmholtz equation. II, Complex Var. Elliptic Equ., 2020, vol. 65, no. 2, pp. 316-332. https://doi.org/10.1080/17476933.2019.1583219.

20. Ergashev T. G. Third double-layer potential for a generalized bi-axially symmetric Helmholtz equation, Ufa Math. J., 2018, vol.10, no.4, pp. 111-121. https://doi.org/ 10.13108/2018-10-4-111.

21. Ehrgashev T. G. The fourth double-layer potential for a generalized bi-axially symmetric Helmholtz equation, Tomsk State University Journal of Mathematics and Mechanics, 2017, no. 50, pp. 45-56 (In Russian).

22. Mukhlisov F. G., Nigmetzyanova A. M. Solution of boundary-value problems for a degenerating elliptic equation of the second kind by the method of potentials, Russian Math. (Iz. VUZ), 2009, vol. 53, no. 8, pp. 46-57. https://doi.org/10.3103/S1066369X09080088.

23. Ergashev T. G. Potentials for three-dimensional singular equation and their application to the solving a mixed problem, Lobachevskii J. Math., 2020, vol.41, no.6, pp. 1067-1077. https://doi.org/10.1134/S1995080220060086.

24. Bateman H., Erdélyi A. Vysshie transtsendentnye funktsii [Higher Transcendental Functions], vol.1, Gipergeometricheskaia funktsiia. Funktsii Lezhandra [The Hypergeometric Function. Legendre Functions]. Moscow, Nauka, 1973, 296 pp. (In Russian)

25. Gradshtein I. S., Ryzhik I. M. Tablitsy integralov, summ, riadov i proizvedenii [Table of Integrals, Series, and Products]. Moscow, Fizmatlit, 1962, 1100 pp. (In Russian)

26. Sabitov K. B. Uravneniia matematicheskoi fiziki [Equations of Mathematical Physics]. Moscow, Fizmatlit, 2013, 352 pp. (In Russian) 